\title{
New evolutionary models for massive ZZ Ceti stars. I. First results for their pulsational properties
}

\author{
L. G. Althaus ${ }^{1, \star}$, A. M. Serenelli ${ }^{1, \star \star}$, A. H. Córsico ${ }^{1, \star \star \star}$, and M. H. Montgomery ${ }^{2}$ \\ ${ }^{1}$ Facultad de Ciencias Astronómicas y Geofísicas, Universidad Nacional de La Plata, Paseo del Bosque S/N, 1900 La Plata, \\ Argentina \\ Instituto de Astrofísica de La Plata, IALP, CONICET \\ e-mail: serenell, acorsico@fcaglp.unlp.edu . ar, mikemon@ast. cam. ac.uk \\ 2 Institute of Astronomy, Madingley Road, Cambridge CB3 OHA, UK
}

Received 7 November 2002 / Accepted 24 March 2003

\begin{abstract}
We present new and improved evolutionary calculations for carbon-oxygen white dwarf (WD) stars appropriate for the study of massive ZZ Ceti stars. To this end, we follow the complete evolution of massive WD progenitors from the zero-age main sequence through the thermally pulsing and mass loss phases to the WD regime. Abundance changes are accounted for by means of a full coupling between nuclear evolution and time-dependent mixing due to diffusive overshoot, semiconvection and salt fingers. In addition, time-dependent element diffusion for multicomponent gases has been considered during the WD stage. Emphasis is placed on the chemistry variations along the whole evolution. In particular, we find that before the ZZ Ceti stage is reached, element diffusion has strongly smoothed out the chemical profile to such a degree that the resulting internal abundance distribution does not depend on the occurrence of overshoot episodes during the thermally pulsing phase. The mass of the hydrogen envelope left at the ZZ Ceti domain amounts to $M_{\mathrm{H}} \approx 2.3 \times 10^{-6} M_{\odot}$. This is about half as large as for the case when element diffusion is neglected. The implications of our new models for the pulsational properties of massive ZZ Ceti stars are discussed. In this regard, we find that the occurrence of core overshooting during central helium burning leaves strong imprints on the theoretical period spectrum of massive ZZ Ceti stars. Finally, we present a simple new prescription for calculating the $\mathrm{He} / \mathrm{H}$ profile which goes beyond the trace element approximation.
\end{abstract}

Key words. stars: evolution - stars: abundances - stars: AGB stars: interiors - stars: white dwarfs - stars: oscillations

\section{Introduction}

Variable DA white dwarf (WD) stars or ZZ Ceti stars belong currently to one of the best established and most extensively studied class of non-radial pulsating stars (see Gautschy \& Saio 1995, 1996 for a review). These hydrogen-rich, pulsating WDs exhibit multiperiodic luminosity variations caused by gravitymodes of low harmonic degree $(\ell \leq 2)$ and periods in the range of $100-1200 \mathrm{~s}$. Such modes were first thought to be excited by the $\kappa$-mechanism (Winget et al. 1981). However, the dominance of convective energy transport in the driving region led Brickhill (1991) and Goldreich \& Wu (1999) to propose convective driving as the main driving mechanism for g-mode oscillations in the DA WDs. ZZ Ceti stars are well known to pulsate in a narrow effective temperature $\left(T_{\text {eff }}\right)$ interval ranging from $10700 \mathrm{~K} \lesssim T_{\text {eff }} \lesssim 12500 \mathrm{~K}$. Numerous important

Send offprint requests to: L. G. Althaus,

e-mail: althaus@fcaglp.fcaglp.unlp.edu.ar

* Member of the Carrera del Investigador Científico y Tecnológico, CONICET, Argentina.

$\star \star$ Fellow of CONICET, Argentina.

$\star \star \star$ Fellow of CONICET, Argentina. studies have been devoted to exploring the pulsational properties of ZZ Ceti stars, amongst them Tassoul et al. (1990), Brassard et al. (1991, 1992a,b), Gautschy et al. (1996) and Bradley (1996, 1998, 2001).

Over the last past decade, the study of the pulsational pattern of variable WDs through asteroseismological techniques has become a very powerful tool for probing the internal structure and evolution of these stars. Indeed, asteroseismological inferences have provided independent valuable constraints to fundamental properties such as core composition, outer layer chemical stratification and stellar mass (Pfeiffer et al. 1996; and Bradley 1998, 2001 amongst others). In particular, asteroseismology of massive ZZ Ceti stars has recently drawn the attention of researchers in view of the possibility it offers to place constraints on the crystallization process in the interior of WDs (Montgomery \& Winget 1999). This has been motivated by the discovery of pulsations in the star BPM 37093 (Kanaan et al. 1992), a massive ZZ Ceti star which should be largely crystallized (Winget el al. 1997).

Needless to say, a detailed WD modeling as well as a complete and self-consistent treatment of the evolutionary stages 
prior to the WD formation are required for an adequate interpretation of the pulsational patterns. In this regard, the outer chemical stratification, the relics of the nucleosynthesis and mixing processes which occurred along the asymptotic giant branch (AGB) evolution (see D'Antona \& Mazzitelli 1990), is a matter of the utmost importance as far as asteroseismology of ZZ Ceti stars is concerned. The construction of ZZ Ceti models based on full evolutionary calculations has recently been undertaken. Indeed, Althaus et al. (2002) have presented detailed models for a $0.563-M_{\odot} \mathrm{ZZ}$ Ceti remnant based on the complete evolution of an initially $3 M_{\odot}$ star, using models which take into account the chemical evolution during the WD regime caused by time-dependent element diffusion. The exploration of the pulsational properties of such models has been performed by Córsico et al. (2001, 2002), who have found that mode trapping effects are considerably weakened as a result of the smoothness of the diffusion-modeled chemical profiles. These studies constitute an improvement as compared with most of the existing research in pulsating DA WDs which invokes diffusive equilibrium in the trace element approximation to assess the shape of hydrogen-helium chemical interface.

In this paper we extend the calculations presented in Althaus et al. (2002) to the case of massive intermediate-mass stars. As compared with Althaus et al. (2002) major improvements in the treatment of the abundance changes have been made. In particular, we developed a time-dependent scheme that fully couples abundance changes due to nuclear burning, mixing processes and element diffusion. Time-dependent mixing due to semiconvection and salt finger is fully taken into account as well as exponentially decaying diffusive overshooting above and below any formally convective region. In particular, the presence of some overshooting below the convective envelope during the thermal pulses has been shown by Herwig et al. (1997) to yield third dredge-up and carbon-rich AGB stars for relatively low initial mass progenitors (see also Ventura et al. 1999; Mazzitelli et al. 1999). In addition, the occurrence of extra mixing below the helium-flash convection zone during the thermally pulsing AGB phase is supported by recent evolutionary calculations (Herwig et al. 1999; Herwig 2000). These studies show that the resulting intershell abundances are in agreement with abundance determinations in hydrogendeficient post-AGB remnants such as PG 1159 stars.

The main aim of this work is to present the first results of detailed and complete evolutionary calculations appropriate for the study of massive ZZ Ceti stars. To this end, we follow the evolution of initially $7.5-$ and $6-M_{\odot}$ stars from the zeroage main sequence through the thermally pulsing phase on the AGB to the WD regime. Attention is concentrated on the chemistry variations along the whole evolution. We emphasize in particular the role of time-dependent element diffusion on the chemical abundance distribution at the ZZ Ceti stage. The exploration of the pulsational properties of massive ZZ Ceti stars in the light of our new models is likewise within the scope of this investigation. Next, in Sect. 2, we briefly describe the main physical inputs of the models, particularly our treatment for the chemical evolution and overshooting. In Sect. 3 we present the main evolutionary results. Pulsational results are discussed in Sect. 4. In that section we also present a simple new prescription for calculating the $\mathrm{He} / \mathrm{H}$ profile, which constitutes an improvement over the trace element approximation. Finally, Sect. 5 is devoted to making some concluding remarks.

\section{Input of the models and evolutionary sequences}

Here we describe at some length the main characteristics of our evolutionary code. We restrict ourselves to the main updates in the macrophysics, particularly the treatment of the chemical abundance changes.

General description of the code: The results presented in this work have been obtained with the stellar evolution code LPCODE we employed in our previous works. The code has been developed at La Plata Observatory and it is described in Althaus et al. (2002) and references therein. For the purposes of the present paper, the code has been substantially modified particularly with regard to the treatment of the abundance changes, modifications which will be described later in this section.

Briefly, the code is based on the method of Kippenhahn et al. (1967) for calculating stellar evolution. Envelope integrations from photospheric starting values inward to a fitting outer mass fraction (close to the photosphere) are performed to specify the outer boundary conditions. The independent variable is $\xi=\ln \left(1-M_{r} / M_{*}\right)$ and the dependent variables are: radius $(r)$, pressure $(P)$, luminosity $(l)$ and temperature $(T)$. The following change of variables is considered in LPCODE:

$$
\begin{aligned}
& \theta^{(n+1)}=\theta^{(n)}+\ln \left(1+u_{\theta}\right) \\
& p^{(n+1)}=p^{(n)}+\ln \left(1+u_{p}\right) \\
& x^{(n+1)}=x^{(n)}+\ln \left(1+u_{x}\right) \\
& l^{(n+1)}=l^{(n)}+u_{l}
\end{aligned}
$$

with $u_{\theta}, u_{p}, u_{x}$ and $u_{l}$ being the quantities to be iterated that are given by $u_{\theta}=\frac{\Delta T}{T^{(n)}}, u_{p}=\frac{\Delta P}{P^{(n)}}, u_{x}=\frac{\Delta r}{r^{(n)}}$ and $u_{l}=\Delta l$, where superscripts $n$ and $n+1$ denote the beginning and end of time interval (see Kippenhahn et al. 1967 for definitions). Here, $\theta=\ln T, x=\ln r$ and $p=\ln P$. Thus, the Henyey iteration scheme is applied to the differences in the luminosity, pressure, temperature and radius between the previous and the computed model.

As for the constitutive physics, LPCODE employs OPAL radiative opacities (including carbon- and oxygen-rich compositions) for arbitrary metallicity from Iglesias \& Rogers (1996) and from Alexander \& Ferguson (1994) for the lowtemperature regime. In particular, opacities for various metallicities are required during the WD cooling regime in view of the metallicity gradient that develops in the envelope of the models as a result of gravitational settling. The equation of state for the low-density regime comprises partial ionization for hydrogen and helium compositions, radiation pressure and ionic contributions. For the high-density regime, partially degenerate electrons and Coulomb interactions are also considered. For the WD regime, we include an updated version of the equation of 
state of Magni \& Mazzitelli (1979). In LPCODE crystallization sets in when the ion coupling constant $\Gamma \equiv Z^{2} e^{2} /\langle r\rangle k_{\mathrm{B}} T$ reaches the value 180 . Neutrino emission rates and conductive opacities are taken from the works of Itoh and collaborators (see Althaus et al. 2002). A nuclear network of 34 thermonuclear reaction rates and 16 isotopes has been considered to describe the hydrogen (proton-proton chain and $\mathrm{CNO}$ bi-cycle) and helium burning, and carbon ignition. Nuclear reaction rates are taken from Caughlan \& Fowler (1988), except for the reactions ${ }^{15} \mathrm{~N}(p, \gamma){ }^{16} \mathrm{O},{ }^{15} \mathrm{~N}(p, \alpha){ }^{12} \mathrm{C},{ }^{18} \mathrm{O}(p, \alpha){ }^{15} \mathrm{~N},{ }^{18} \mathrm{O}(p, \gamma){ }^{19} \mathrm{~F}$, ${ }^{12} \mathrm{C}(\alpha, \gamma){ }^{16} \mathrm{O},{ }^{16} \mathrm{O}(\alpha, \gamma){ }^{20} \mathrm{Ne},{ }^{13} \mathrm{C}(\alpha, n){ }^{16} \mathrm{O},{ }^{18} \mathrm{O}(\alpha, \gamma){ }^{22} \mathrm{Ne}$, ${ }^{22} \mathrm{Ne}(\alpha, n)^{25} \mathrm{Mg}$ and ${ }^{22} \mathrm{Ne}(\alpha, \gamma)^{26} \mathrm{Mg}$, which are taken from Angulo et al. (1999). In particular, the $\left.{ }^{12} \mathrm{C}(\alpha, \gamma)\right)^{16} \mathrm{O}$ reaction rate given by Angulo et al. (1999) is about twice as large as that of Caughlan \& Fowler (1988).

To get a reasonable numerical accuracy, AGB models typically contained 1400 mesh points, except for the peak of the thermal pulses where about 2000 mesh points were required. Evolutionary time steps during the thermally pulsing phase ranged from a few days during the helium flashes and the subsequent phases where the third dredge-up may occur to some years during the stationary hydrogen-burning interpulse phase. Finally, mesh distribution is performed every three time steps.

Chemical evolution: An important aspect of the present study is the modeling of the chemical abundance distribution throughout all of the different evolutionary phases. To this end, we consider a time-dependent scheme for the simultaneous treatment of chemical changes caused by nuclear burning and convective, semiconvective, salt finger and overshoot mixing. Needless to say, such a coupling between nuclear evolution and timedependent mixing is much more physically sound than the instantaneous mixing approximation usually assumed in stellar modeling. In particular, this kind of treatment has been used by Mazzitelli et al. (1999) to study the lithium production by hot bottom burning in AGB stars (see also Ventura et al. 1999).

In what follows, we present some details about the numerical method for the abundance changes included in LPCODE. Specifically, the abundance changes for all chemical elements are described by the set of equations

$\left(\frac{\mathrm{d} \boldsymbol{Y}}{\mathrm{d} t}\right)=\left(\frac{\partial \boldsymbol{Y}}{\partial t}\right)_{\mathrm{nuc}}+\frac{\partial}{\partial M_{r}}\left[\left(4 \pi r^{2} \rho\right)^{2} D \frac{\partial \boldsymbol{Y}}{\partial M_{r}}\right]$

with $\boldsymbol{Y}$ being the vector containing the number fraction of all considered nuclear species. Here, mixing due to convection, semiconvection, salt finger and overshoot is treated as a diffusion process which is described by the second term of Eq. (2) in terms of the mass coordinate $M_{r}$. The efficiency of mixing processes is described by appropriate diffusion coefficients $D$ (see later in this section). The first term of Eq. (2) gives the abundance changes due to thermonuclear reactions, changes which, in the present work, are fully coupled to mixing processes. This term is expanded as a function of local abundances and cross sections following the implicit scheme by Arnett \& Truran (1969). After linearization, it can be written as

$\left(\frac{\boldsymbol{Y}_{j}^{n+1}-\boldsymbol{Y}_{j}^{n}}{\Delta t}\right)_{\mathrm{nuc}}=-\Gamma_{j} \boldsymbol{Y}^{n+1}+\boldsymbol{\Lambda}_{j}$

where $\Delta t$ is the time interval $\Delta t=t^{n+1}-t^{n}$ and superscripts $n$ and $n+1$ again denotes the beginning and end of time interval. Subscript $j$ denotes the $j$ th mesh point. $\Gamma_{j}$ is a $N \times N$ matrix ( $N$ being the number of nuclear species considered) and $\Lambda_{j}$ a vector of $N$ dimension, both with elements involving abundances $\left(\boldsymbol{Y}_{j}^{n}\right)$ and nuclear reaction rates at $t^{n}$. Using a three-point, finite-difference scheme, the second term of Eq. (2) can be approximated by

$\left\{\frac{\partial}{\partial M_{r}}\left[\left(4 \pi r^{2} \rho\right)^{2} D \frac{\partial \boldsymbol{Y}}{\partial M_{r}}\right]\right\}_{j}^{n+1}=\mathcal{A} \boldsymbol{Y}_{j-1}^{n+1}+\mathcal{B} \boldsymbol{Y}_{j}^{n+1}+C \boldsymbol{Y}_{j+1}^{n+1}$

Here $\mathcal{A}, \mathcal{B}$ and $\mathcal{C}$ are diagonal matrices with dimension $N \times N$. Specifically, the non-vanishing elements of such matrices are given by

$a_{i i}=\frac{(4 \pi)^{2}\left(\rho^{2} r^{4} D\right)_{j-1 / 2}}{\left(m_{j-1 / 2}-m_{j+1 / 2}\right)\left(m_{j-1}-m_{j}\right)}$

$c_{i i}=\frac{(4 \pi)^{2}\left(\rho^{2} r^{4} D\right)_{j+1 / 2}}{\left(m_{j-1 / 2}-m_{j+1 / 2}\right)\left(m_{j}-m_{j+1}\right)}$

$b_{i i}=-\left(a_{i i}+c_{i i}\right)$

where the subscripts $j+1 / 2(j-1 / 2)$ indicate an average for the mass shell between the mesh-points $j$ and $j+1(j-1)$. At the boundaries of the mass interval of integration, we have no mass flux $\left(\partial \boldsymbol{Y} / \partial M_{r}=0\right)$, thus

$\frac{\partial}{\partial M_{r}}\left[\left(4 \pi r^{2} \rho\right)^{2} D \frac{\partial \boldsymbol{Y}}{\partial M_{r}}\right]_{\mathrm{bdry}}=\left(4 \pi r^{2} \rho\right)^{2} D \frac{\partial^{2} \boldsymbol{Y}}{\partial M_{r}^{2}}$

A second-order Taylor expansion in the abundances leads us to

$c_{i i}=2 \frac{(4 \pi)^{2}\left(\rho^{2} r^{4} D\right)_{1 / 2}}{\left(m_{1}-m_{2}\right)^{2}} ; \quad b_{i i}=-c_{i i} ; \quad a_{i i}=0$

at the top boundary, and to

$a_{i i}=2 \frac{(4 \pi)^{2}\left(\rho^{2} r^{4} D\right)_{J-1 / 2}}{\left(m_{J-1}-m_{J}\right)^{2}} ; \quad b_{i i}=-a_{i i} ; \quad c_{i i}=0$

at the bottom boundary (characterized by the $J$ th mesh-point).

Equations (2)-(4) lead to the following system of linear equations to be solved simultaneously for the new chemical abundances $\boldsymbol{Y}^{n+1}$ at time $t^{n+1}$ :

$\mathcal{F}_{j} \boldsymbol{Y}_{j}^{n+1}-\mathcal{A}_{j} \boldsymbol{Y}_{j-1}^{n+1}-\mathcal{C}_{j} \boldsymbol{Y}_{j+1}^{n+1}=\mathcal{M}_{j}$

where $\mathcal{F}_{j}=\mathcal{T}^{-1}+\Gamma_{j}-\mathcal{B}_{j}$ and $\boldsymbol{M}_{j}=\boldsymbol{\Lambda}_{j}+\boldsymbol{Y}_{j}^{n} / \Delta t . \mathcal{T}^{-1}$ is a $N \times N$ diagonal matrix with elements ${ }^{1} 1 / \Delta t$. Fortunately, Eq. (11) has a special structure (tridiagonal) which enables us to minimize

1 Note that Eq. (11) couples nuclear evolution to the current composition change due to mixing processes. 
storage of matrix coefficients. Schematically, we have $\left(\mathcal{A}_{j}, \mathcal{F}_{j}\right.$ and $C_{j}$ are matrices and $\boldsymbol{Y}_{j}^{n+1}$ and $\mathcal{M}_{j}$ are vectors)

$$
\left(\begin{array}{ccccccccc}
\mathcal{F}_{1} & \mathcal{C}_{2} & & & & & & & \\
\mathcal{A}_{1} & \mathcal{F}_{2} & C_{3} & & & & & & \\
& \mathcal{A}_{2} & \mathcal{F}_{3} & C_{4} & & & & & \\
& & \ddots & \ddots & \ddots & & & & \\
& & & \ddots & \ddots & \ddots & & & \\
& & & & \ddots & \ddots & \ddots & & \\
& & & & \mathcal{A}_{J-3} & \mathcal{F}_{J-2} & C_{J-1} & \\
& & & & & \mathcal{A}_{J-2} & \mathcal{F}_{J-1} & C_{J} \\
& & & & & & \mathcal{A}_{J-1} & \mathcal{F}_{J}
\end{array}\right) \bullet
$$$$
\text { •(c) } \left.\begin{array}{c}
\boldsymbol{Y}_{1}^{n+1} \\
\boldsymbol{Y}_{2}^{n+1} \\
\boldsymbol{Y}_{3}^{n+1} \\
\vdots \\
\vdots \\
\vdots \\
\boldsymbol{Y}_{J-2}^{n+1} \\
\boldsymbol{Y}_{J-1}^{n+1} \\
\boldsymbol{Y}_{J}^{n+1}
\end{array}\right)=\left(\begin{array}{c}
\mathcal{M}_{1} \\
\mathcal{M}_{2} \\
\mathcal{M}_{3} \\
\vdots \\
\vdots \\
\vdots \\
\mathcal{M}_{J-2} \\
\mathcal{M}_{J-1} \\
\mathcal{M}_{J}
\end{array}\right) .
$$

To solve this set of equations, we followed the method considered by Iben \& MacDonald (1985). Equation (2) is applied to radiative $(D=0)$ zones and to convective, semiconvective, overshoot and salt finger regions provided the diffusion coefficient $D$ is appropriately specified for each process according to the adopted treatment of convection. We consider a total of 16 isotopes $(N=16):{ }^{1} \mathrm{H},{ }^{2} \mathrm{H},{ }^{3} \mathrm{He},{ }^{4} \mathrm{He},{ }^{7} \mathrm{Li},{ }^{7} \mathrm{Be},{ }^{12} \mathrm{C},{ }^{13} \mathrm{C}$, ${ }^{14} \mathrm{~N},{ }^{15} \mathrm{~N},{ }^{16} \mathrm{O},{ }^{17} \mathrm{O},{ }^{18} \mathrm{O},{ }^{19} \mathrm{~F},{ }^{20} \mathrm{Ne}$ and ${ }^{22} \mathrm{Ne}$. In LPCODE, abundance changes are performed after convergence of each stellar model (and not during iterations). For a better integration precision in the nuclear evolution computation, each evolutionary time step is divided into 5 chemical time steps.

The evolution of the chemical abundance distribution caused by element diffusion during the whole WD evolution constitutes an important point of the present work. In our treatment of time-dependent diffusion we have considered gravitational settling and chemical and thermal diffusion for the following nuclear species: ${ }^{1} \mathrm{H},{ }^{3} \mathrm{He},{ }^{4} \mathrm{He},{ }^{12} \mathrm{C},{ }^{14} \mathrm{~N}$ and ${ }^{16} \mathrm{O}$. The chemical evolution resulting from element diffusion is described, for a given isotope $i$, by the continuity equation as

$\left(\frac{\partial Y_{i}}{\partial t}\right)_{\mathrm{diff}}=-\frac{\partial}{\partial M_{r}}\left(4 \pi r^{2} \rho Y_{i} w_{i}\right)$,

where $w_{i}$ is the diffusion velocity. We have adopted the treatment of Burgers (1969) for multicomponent gases. In this work, our focus is on the chemical evolution occurring quite deep in the star, thus radiative levitation (which is expected to modify the surface composition of hot WDs) has been neglected. In terms of the gradient of ion densities, diffusion velocities can be written in the form

$w_{i}=w_{i}^{\mathrm{gt}}-\sum_{\operatorname{ions}(j)} \sigma_{i j} \frac{\mathrm{d} \ln n_{j}}{\mathrm{~d} r}$, where $w_{i}^{\mathrm{gt}}$ stands for the velocity component due to gravitational settling and thermal diffusion. Details are given in Althaus \& Benvenuto (2000); see also Gautschy \& Althaus (2002) for an application to DB WDs. Unlike our previous works, the abundance changes resulting from element diffusion are fully coupled to nuclear reactions. To this end, matrix coefficients in Eq. (12) have been appropriately modified.

Overshooting: In the present study we have included timedependent overshoot mixing during all pre-WD evolutionary stages. The scheme for the abundance changes described above enables us a self-consistent treatment of diffusive overshooting in the presence of nuclear burning. In particular, we have considered exponentially decaying diffusive overshooting above and below any formally convective region, including the convective core (main sequence and central helium burning phases), the external convective envelope and the shortlived helium-flash convection zone which develops during the thermal pulses. Specifically, we have followed the formalism of Herwig (2000) based on the hydrodynamical simulations of Freytag et al. (1996), which show that turbulent velocities decay exponentially outside the convective boundaries. The expression for the diffusion coefficient in overshoot regions is

$D_{\mathrm{os}}=D_{0} \exp \left(\frac{-2 z}{H_{\mathrm{v}}}\right)$

where $D_{0}$ is the diffusion coefficient at the boundary of the convection zone (see below), $z$ is the radial distance from the edge of the convection zone, $H_{\mathrm{v}}=f H_{\mathrm{P}}$, where the free parameter $f$ is a measure of the extent of the overshoot region, and $H_{\mathrm{P}}$ is the pressure scale height at the convective boundary. In this study we have assumed $f=0.015$, which accounts for the observed width of the main sequence as well as the intershell abundances of hydrogen-deficient post-AGB remnants (see Herwig et al. 1997, 1999; Herwig 2000 for details; see also Mazzitelli et al. 1999).

Treatment of convection: For this work, we included in LPCODE the extended mixing length theory of convection for fluids with composition gradients developed by Grossman et al. (1993) in its local approximation as given by Grossman $\&$ Taam (1996). These authors have developed the non-linear mixing length theory of double diffusive convection that applies in convective, semiconvective and salt finger instability regimes. According to this treatment, the diffusion coefficient $D$ in Eq. (2) characterizing such mixing regimes is given by

$D=\ell \sigma$

where $\ell=\alpha H_{P}$ is the mixing length and $\sigma$ the turbulent velocity. The value of $\sigma$ is determined by simultaneously solving the equations for the turbulent velocity and flux conservation (Eqs. (9) and (17) of Grossman \& Taam 1996). In this work, the free parameter $\alpha$ is taken to be 1.5 . 
Mass loss: Our treatment of mass loss is that of Blöcker (1995). In particular, during the AGB evolution, the mass loss rate is given by

$\dot{M}=4.83 \times 10^{-9} M_{\mathrm{ZAMS}}^{-2.1} L^{2.7} \dot{M}_{\mathrm{R}}\left[M_{\odot} / \mathrm{yr}\right]$

where $\dot{M}_{\mathrm{R}}$ is the usual Reimer rate given by $\dot{M}_{\mathrm{R}}=$ $4 \times 10^{-13} \eta_{\mathrm{R}} L R / M$ with $L, R$ and $M$ the luminosity, radius and mass of the star in solar units. This formulation, based on dynamical computations for atmospheres of Mira-like stars, accounts for the strong increase of the mass loss rate and modulations by thermal pulses expected in the course of the AGB evolution. We set the value of the free parameter $\eta_{\mathrm{R}}$ somewhat arbitrarily at 1 in order to get a reasonable number of thermal pulses on the AGB. A more careful choice for the value of $\eta_{\mathrm{R}}$ is not relevant for the purposes of the present paper. In our computation, mass loss episodes taking place during the stages of core helium burning and red giant branch are considered according to the usual Reimer's formulation with $\eta_{\mathrm{R}}=1$.

Global properties of the evolutionary computations: A primary target of this work is the construction of detailed massive DA WD models with ${ }^{12} \mathrm{C} /{ }^{16} \mathrm{O}$ cores appropriate for the pulsational studies. Because the self-consistent solution of nuclear evolution and time-dependent mixing demands a considerable increase of computing time, particularly during the AGB phase of the WD progenitor, we restrict ourselves to examining two cases of evolution for the progenitor:

- As for the first case, which will be hereinafter referred to as sequence NOV, we computed the complete evolution of an initially $7.5-M_{\odot}$ stellar model without overshooting. The sequence was followed from the zero-age main sequence all the way from the stages of hydrogen and helium burning in the core up to the thermally pulsing phase at the tip of the AGB. After experiencing several thermal pulses and as a result of mass loss episodes, the progenitor departs from the AGB and evolves towards its WD configuration. Specifically, during the 7 th pulse, an enhanced mass loss rate is invoked to terminate the AGB phase. Evolution was pursued to the domain of the ZZ Ceti stars on the WD cooling branch. The final mass of the WD remnant is about $0.936 M_{\odot}$.

- As for the second case (sequence OV), we followed the evolution of a $6-M_{\odot}$ progenitor from the main sequence until the star underwent four thermal pulses. Here, diffusive overshooting was applied during all evolutionary stages and to all convective boundaries. This sequence was done with the aim of assessing the influence of overshooting on the WD internal chemical stratification and to explore its implications for the pulsational properties of massive ZZ Ceti stars as well. For this sequence, we have not computed the post-AGB evolution. Rather, we obtained an initial WD configuration by simply scaling the chemical profile builtup during the pre-WD evolution to the structure of a hot WD model resulting from sequence NOV. Because we are interested in the final chemical stratification of $\mathrm{ZZ}$ Ceti models, this procedure is enough for our purposes. Here, the mass of the ${ }^{12} \mathrm{C} /{ }^{16} \mathrm{O}$ core towards the end of thermal pulses amounts to $0.94 M_{\odot}$.

For both sequences, a solar-like initial composition $(Y, Z)=$ $(0.275,0.02)$ corresponding to Anders \& Grevesse (1989) has been adopted. Interestingly, the mass of the resulting ${ }^{12} \mathrm{C} /{ }^{16} \mathrm{O}$ core is quite similar in both sequences despite the very different initial mass values of the progenitors (see also Mazzitelli et al. 1999). In fact, overshooting leads to larger convective cores during the core burning stages. We want to mention that the breathing pulse instability occurring towards the end of core helium burning has been suppressed (see Straniero et al. 2003 for a recent discussion about this point). Finally, for both sequences, time-dependent element diffusion was considered during the whole WD evolution.

To the best of our knowledge, this is the first time that WD models appropriate for the study of pulsational properties of massive ZZ Ceti stars are derived from detailed evolutionary calculations which include a self-consistent treatment of timedependent element diffusion and nuclear burning. We report below the predictions of our calculations, particularly for the chemical stratification and comment on their implications for the relevant pulsation properties.

\section{Evolutionary results}

\subsection{Evolution of the white dwarf progenitor}

In this section, we describe the main results for the evolutionary stages prior to the WD formation. Attention will be restricted to analysing the relevant aspects for the WD formation, in particular the chemistry variations along the evolution. Although the full coupling between nuclear evolution and mixing implemented in LPCODE is appropriate for addressing problems such as hot bottom burning and lithium production in AGB stars, we will not explore them in this paper. Such specific issues would carry us too far afield, and we refer the reader to the works of D'Antona \& Mazzitelli (1996) and Mazzitelli et al. (1999) for details. Other recent relevant studies of AGB evolution are those of Sackmann \& Boothroyd (1992), Vassiliadis \& Wood (1993), Blöcker (1995), Straniero et al. (1997), Wagenhuber \& Groenewegen (1998) and Herwig (2000) amongst others.

We begin by examining Fig. 1 which illustrates the Hertzsprung-Russell (HR) diagram for the WD progenitor from the main sequence to an advanced AGB phase. Solid and dashed lines correspond to sequences OV and NOV, respectively. A feature worthy of comment predicted by sequence $\mathrm{OV}$ is that central helium burning occurs mostly during a loop to the blue in the HR diagram. Indeed, the blue excursion begins when the central helium abundance by mass falls below $\approx 0.75$ and continues until the abundance has decreased below 0.1 . For this sequence, the total time spent during hydrogen and helium burning in the core amounts to $7.5 \times 10^{7} \mathrm{yr}$, while for sequence NOV this time is $4.2 \times 10^{7} \mathrm{yr}$. Following the exhaustion of central helium and before the re-ignition of the hydrogen burning shell both of our sequences experience the second dredge-up. As a result, the surface composition is appreciably modified, 


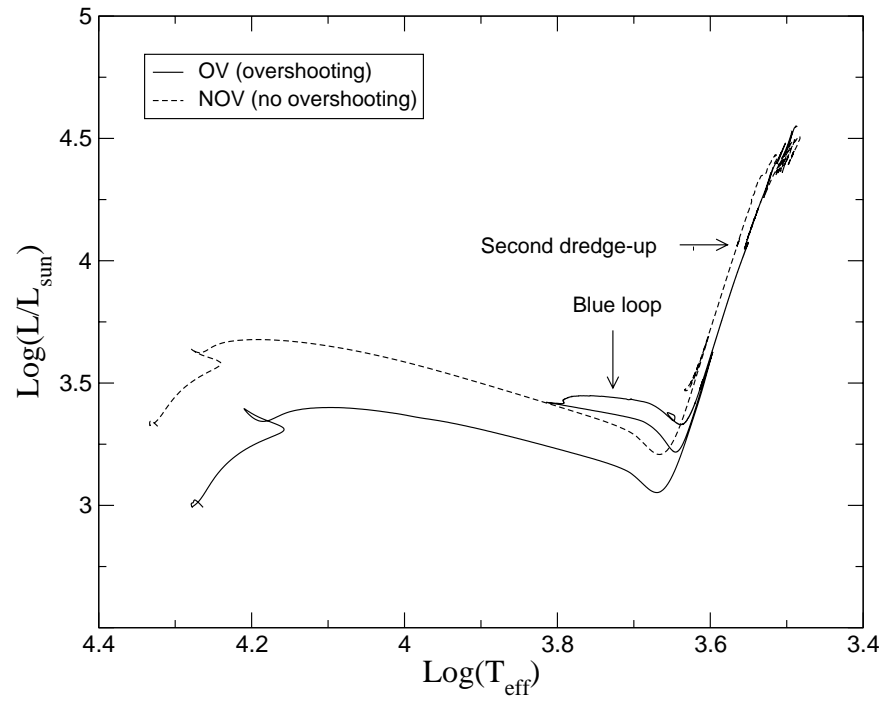

Fig. 1. The Hertzsprung-Russell diagram for the evolution of our 7.5and $6-M_{\odot}$ sequences (NOV and OV, respectively) from the main sequence to the first thermal pulses.

particularly for sequence OV. In fact, for this sequence, the envelope helium abundance rises from about 0.30 (resulting from the first dredge-up) to 0.364 . For sequence NOV, the second dredge-up brings the helium abundance to 0.328 . It is also worth mentioning that during the second dredge-up phase, the mass of the hydrogen-exhausted core is strongly reduced. In particular, for sequence NOV, the core mass is reduced from $\approx 1.50 M_{\odot}$ at the end of central helium burning to $\approx 0.93 M_{\odot}$ after the dredge-up episode. For sequence OV, the hydrogenexhausted core is reduced from 1.38 to $0.938 M_{\odot}$ during the dredge-up.

The inner chemistry variations that take place along the red giant branch and early AGB evolution is well documented in Figs. 2 and 3 for sequence NOV and OV, respectively. Specifically, we show the evolution of the internal helium, carbon and oxygen distribution as a function of mass for the evolutionary stages following the end of helium burning in the core. We begin by examining the results for sequence NOV depicted in Fig. 2. The upper panel shows the chemical profile when the helium convective core vanishes leaving a central oxygen abundance of 0.55 by mass. As evolution proceeds, the helium-rich layers overlying the former convective core are radiatively burnt, giving rise to an off-centred peak in the carbon and oxygen abundances. This is shown by the middle panel of Fig. 2, which corresponds to the moment when the star surface luminosity exceeds $\log \left(L / L_{\odot}\right)=3.72$ for the first time after $4.25 \times 10^{7} \mathrm{yr}$ of evolution. Because the oxygen abundance, and therefore the mean molecular weight, decreases inwards at $M_{r} \approx 0.45 M_{\odot}$, a salt finger instability characterized by a large diffusion coefficient develops at this point. The resulting salt finger mixing is responsible for the redistribution of the innermost ${ }^{12} \mathrm{C} /{ }^{16} \mathrm{O}$ profile, redistribution that takes place during the following $3 \times 10^{5} \mathrm{yr}$ and which is documented by the bottom panel of Fig. 2. Note also that during this time interval the mass of the ${ }^{12} \mathrm{C} /{ }^{16} \mathrm{O}$ core has increased considerably by virtue of helium shell burning, reaching $0.925 M_{\odot}$ by the re-ignition of the

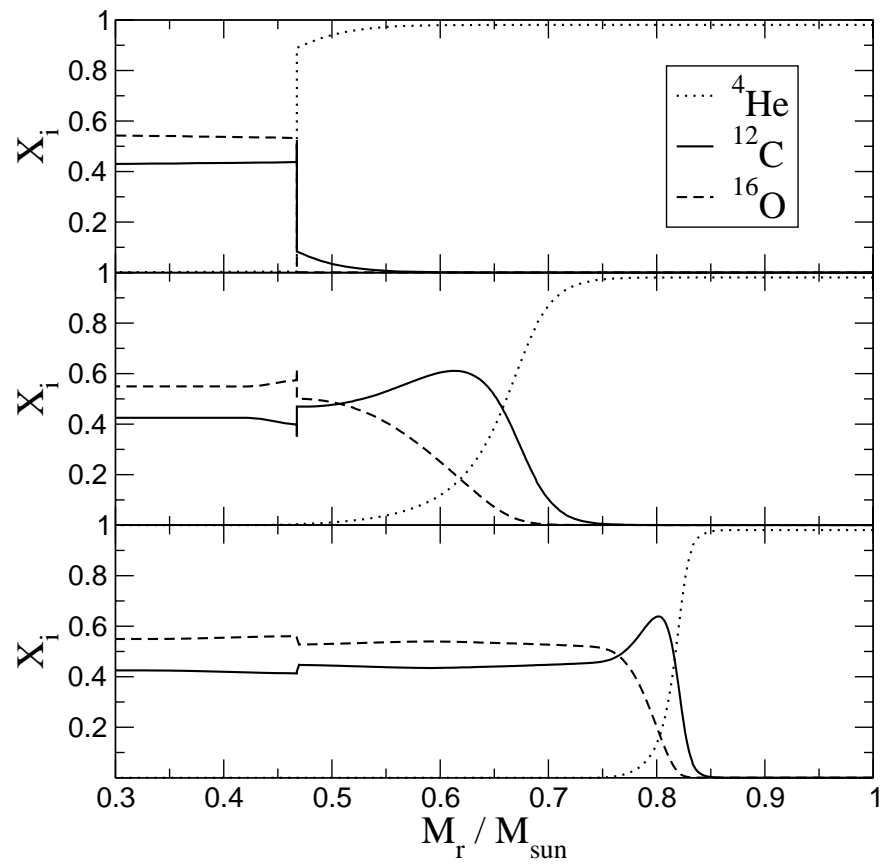

Fig. 2. Internal ${ }^{4} \mathrm{He},{ }^{12} \mathrm{C}$ and ${ }^{16} \mathrm{O}$ profiles for the $7.5-M_{\odot}$ sequence (NOV) at three evolutionary stages after the end of core helium burning. A salt finger instability develops at $M_{r} \approx 0.45 M_{\odot}$ (middle panel) which leads to a chemical redistribution there (bottom panel). See text for details.

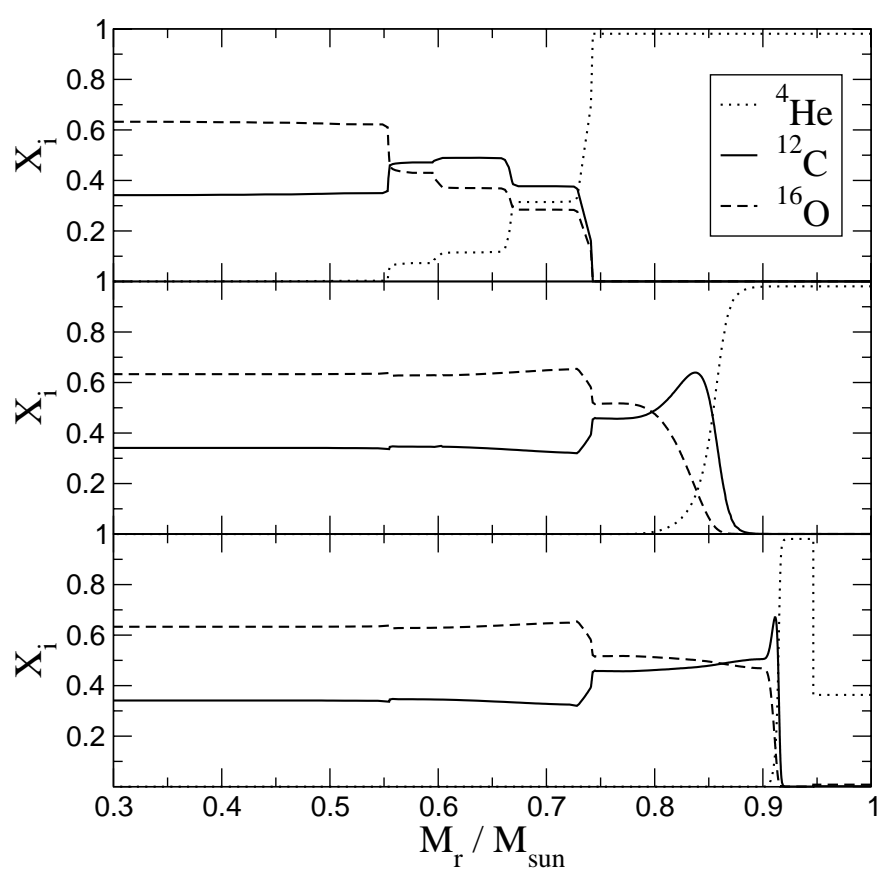

Fig. 3. Same as Fig. 2 but for the $6-M_{\odot}$ sequence $(\mathrm{OV})$. Core overshooting leads to a larger ${ }^{12} \mathrm{C} /{ }^{16} \mathrm{O}$ core by the end of central helium burning.

hydrogen shell before the occurrence of the first helium thermal pulse. The behaviour for sequence OV is detailed in Fig. 3. Here, the size of the ${ }^{12} \mathrm{C} /{ }^{16} \mathrm{O}$ core emerging from the convective helium core burning (upper panel) becomes substantially larger (we remind the reader that sequence OV has a lower initial stellar mass than sequence NOV). Thus, a smaller fraction 


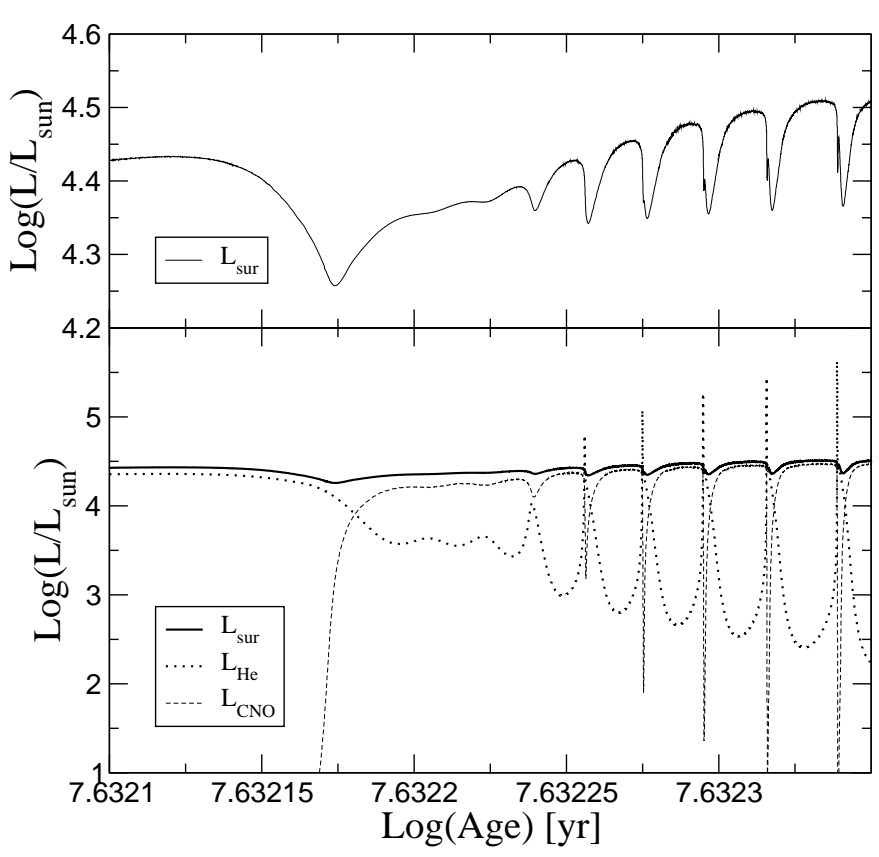

Fig. 4. Upper panel: the time-dependence of surface luminosity (in solar units) for the 7.5- $M_{\odot}$ sequence (NOV) during the first thermal pulses at the AGB. Bottom panel: the time-dependence of surface luminosity, hydrogen- (as given by CNO cycle reactions) and heliumburning luminosities (dashed and dotted lines respectively) for the same sequence as above. The time-scale is given in $\mathrm{yr}$ from the main sequence. A total of 7 thermal pulses have been computed before the WD progenitor departs from the AGB as a result of mass loss episodes.

of helium remains unburned and so a shorter pre-AGB phase is expected. In fact, about $4.2 \times 10^{5} \mathrm{yr}$ are needed to evolve from the end of core helium burning to the onset of the thermally pulsing AGB phase (bottom panel), as compared with the $9.3 \times 10^{5} \mathrm{yr}$ for sequence NOV. The carbon and oxygen distribution in the core is clearly different from the case without overshooting, a feature which is expected to bear its signature in the theoretical period spectrum of ZZ Ceti stars (see Sect. 4). Note also that sequence $\mathrm{OV}$ is characterized by a somewhat larger central oxygen abundance ( 0.63 by mass) than expected for sequence NOV. We want to stress again that breathing pulses have been suppressed in our calculations. The suppression of breathing pulses inhibits the formation of ${ }^{12} \mathrm{C} /{ }^{16} \mathrm{O}$ cores with large central oxygen abundances (see Straniero et al. 2003 and references therein). However, when central helium has been substantially depleted, sequence OV experiences a small growth of the convective helium core, which increases the central helium abundance from 0.07 to 0.10 . This fact is, in part, responsible for the higher central oxygen abundance that characterizes sequence OV.

Towards the end of the early AGB phase, hydrogen is reignited in a thin shell and the star begins to thermally pulse. Here, helium shell burning becomes unstable (see Iben \& Renzini 1983 for a detailed description of this phase). The time dependence of surface luminosity, hydrogen- and heliumburning luminosities for sequence NOV during the first five thermal pulses is given in Fig. 4. The interpulse period for this

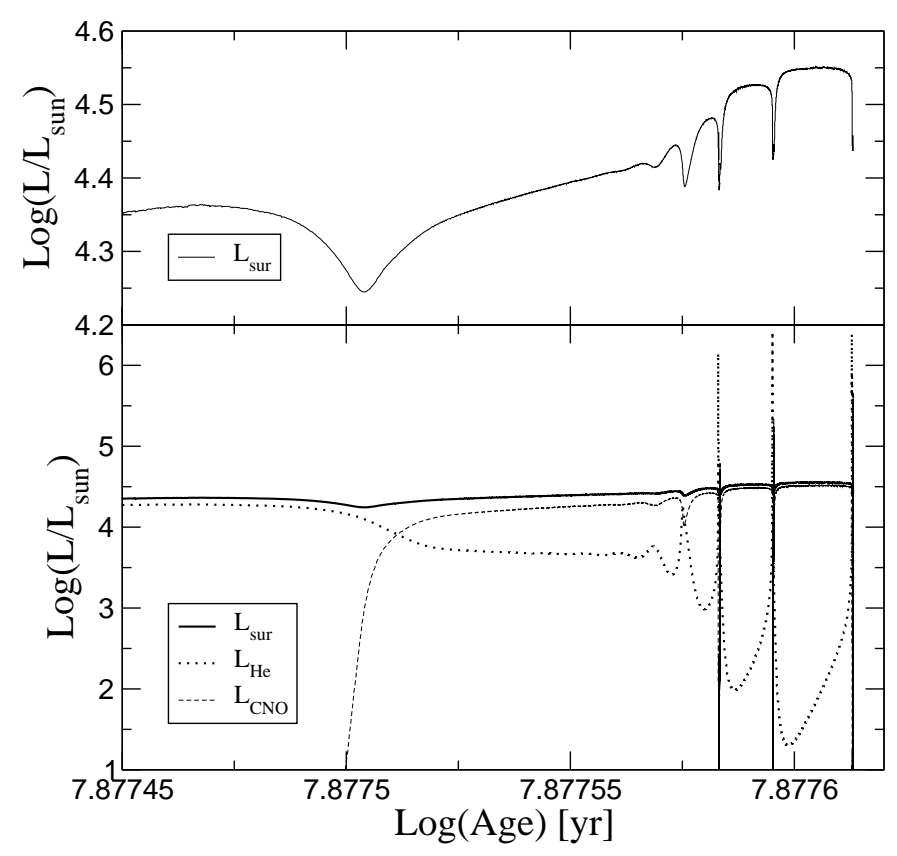

Fig. 5. Same as Fig. 4 but for the $6-M_{\odot}$ sequence $(\mathrm{OV})$. Here, the first three thermal pulses are shown.

sequence is roughly $2.4 \times 10^{3} \mathrm{yr}$. Note that the helium burning rate rises very steeply at each pulse. After experiencing 7 thermal pulses and considerable mass loss, the WD progenitor departs from the AGB and evolves towards the planetary nebula region and eventually to the WD state. In our simulation, when departure from the AGB occurs, stationary helium-shell burning mostly contributes to the star luminosity, but shortly after, hydrogen burning takes over. During the thermal pulses, mass loss episodes reduce the stellar mass from 6.15 to $0.936 M_{\odot}$. It is worth noting that during the interpulses the temperature at the base of the convective envelope becomes high enough for hydrogen-burning reactions to occur. The nuclear processing at the base of the convective envelope is commonly referred to as "hot bottom burning", a process whose occurrence is strongly dependent on the treatment of convection. As we mentioned, we will not discuss this aspect here (see D'Antona \& Mazzitelli 1996; Mazzitelli et al. 1999, for recent studies); suffice it to say that for sequence NOV, temperatures at the bottom of the convective envelope after the first five pulses reach about $6 \times 10^{7} \mathrm{~K}$, which is high enough for an early, albeit moderate, onset of hot bottom burning to occur. In fact, the surface carbon abundance decreases from 0.0021 to 0.0019 during this phase.

The presence of overshooting from below the convective regions affects the evolution during the thermally pulsing AGB phase. The run of luminosities for sequence OV is detailed in Fig. 5. Note that the trend of the luminosity evolution is different from sequence NOV. In particular, the rise in the helium shell burning luminosity at the peak of the pulse is much more noticeable than in sequence NOV. This can be understood on the basis that overshoot from the bottom of the helium-flash convection zone carries some helium from the intershell region into deeper layers, where it is burnt at higher temperatures. This picture can be visualized by examining Fig. 6, in which we show the internal abundance distribution versus mass 


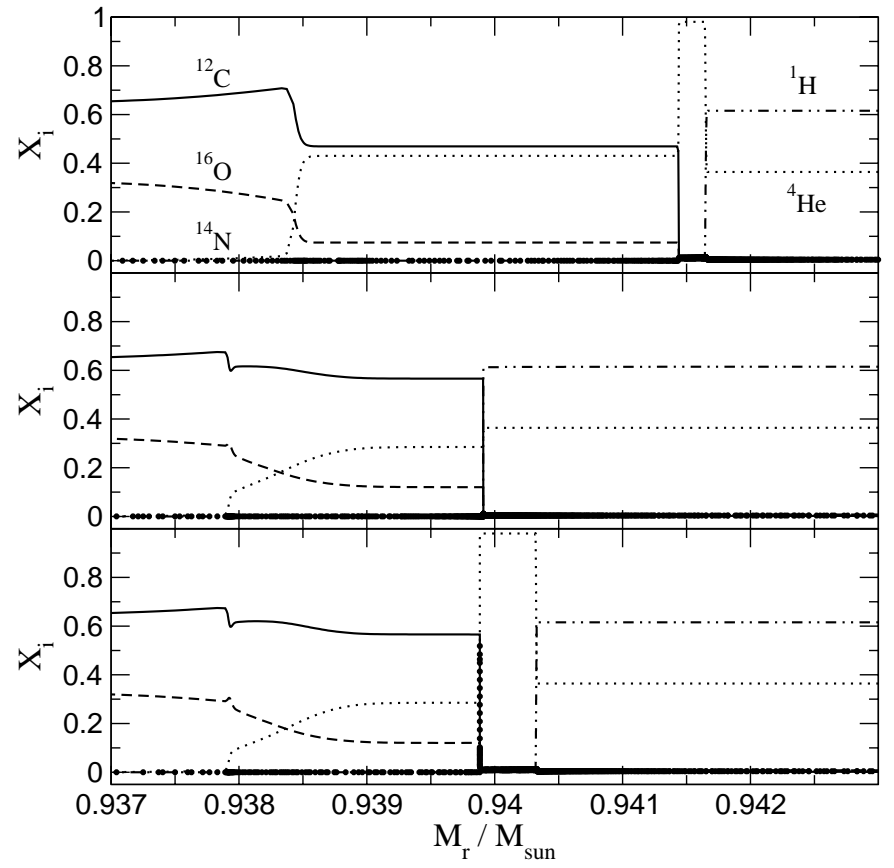

Fig. 6. Chemical profiles for the 6- $M_{\odot}$ sequence $(\mathrm{OV})$ at three evolutionary stages during the 2nd thermal pulse. At pulse peak (upper panel), the model is characterized by a ${ }^{4} \mathrm{He}$-rich buffer and an underlying region rich in ${ }^{4} \mathrm{He},{ }^{12} \mathrm{C}$ and ${ }^{16} \mathrm{O}$ (intershell). The ${ }^{4} \mathrm{He}$-flash convection zone and overshooting extending down to $M_{r} \approx 0.938 M_{\odot}$ are responsible for the redistribution of the intershell abundances (middle panel). Note also that envelope convection has penetrated down to $M_{r}=0.9399 M_{\odot}$, sweeping away the ${ }^{4} \mathrm{He}$ buffer and reaching ${ }^{12} \mathrm{C}$-rich regions (third dredge-up). During the interpulse, the helium buffer is built-up again (bottom panel). Note also the formation of a small ${ }^{14} \mathrm{~N}-$ pocket at the base of the helium buffer.

coordinate during the second thermal pulse of sequence OV. The region illustrated comprises the base of the hydrogen/helium envelope, the almost pure helium buffer, the intershell region and the top of the ${ }^{12} \mathrm{C} /{ }^{16} \mathrm{O}$ core. The upper panel corresponds to the moment at which the model is at the peak of its second thermal pulse. Here, the model is characterized by a short-lived (few years) convection zone, the product of the huge flux of energy caused by unstable helium burning. This convection zone extends from $M_{r} \approx 0.9414 M_{\odot}$ down to the base of the helium-burning region at $0.9388 M_{\odot}$. At the bottom of this helium-flash convection zone there exists an overshoot region stretching down to the underlying carbonrich layers (the ${ }^{12} \mathrm{C} /{ }^{16} \mathrm{O}$ core) at about $0.9383 M_{\odot}$. As a result of this overshoot region, larger amounts of carbon and oxygen are mixed up, as compared with the situation in which overshooting is neglected. This mixing episode is particularly important regarding the outer layer chemical stratification of the emerging WD remnant (see Herwig et al. 1997). As can be seen from the middle panel of Fig. 6, the resulting abundances of the intershell zone are $\left({ }^{4} \mathrm{He} /{ }^{12} \mathrm{C} /{ }^{16} \mathrm{O}\right)=(28 / 56 / 12)$ as compared with $(74 / 23 / 0.4)$ for sequence $\mathrm{NOV}^{2}$. These results quantitatively agree with the predictions of Herwig (2000) for

\footnotetext{
${ }^{2}$ The quoted ${ }^{4} \mathrm{He} /{ }^{12} \mathrm{C}$ values for the intershell abundances change somewhat with subsequent pulses, see Herwig (2000).
}

the case of lower stellar masses than considered here. Note also that envelope convection (and overshoot from below) has penetrated into deeper layers down to $M_{r}=0.9399 M_{\odot}$, reaching ${ }^{12} \mathrm{C}$-rich regions (third dredge-up ${ }^{3}$ ). During this process, the helium buffer, which was built-up by hydrogen shell burning during the preceding pulse, is completely wiped out. These episodes take place while hydrogen shell burning is almost extinct. Sequence OV experiences important dredge-up of carbon even at the first pulse; however, hot bottom burning taking place during the following quiescent interpulse phase is so efficient (with temperatures at the base of the convective envelope exceeding $7 \times 10^{7} \mathrm{~K}$ ) in converting carbon into nitrogen that the formation of a carbon star is avoided (at least during the first thermal pulses). Interestingly, about $25 \%$ of the luminosity of the star during this phase is produced within the convective envelope.

Bottom panel of Fig. 6 shows that the helium buffer is builtup again during the interpulse phase and a next pulse is thus initiated. Finally, another feature predicted by our calculations is the presence of a small radiative ${ }^{14} \mathrm{~N}$-pocket at the base of the helium buffer (see also Herwig 2000 for a similar finding). In fact, during the third dredge-up diffusive overshoot has led to the formation of a small region in which hydrogen from the envelope and carbon from the intershell region coexist in appreciable abundances. When this region heats up enough to reignite hydrogen burning, one of the main results is the formation of ${ }^{14} \mathrm{~N}$ with abundances reaching about 0.5 . However, the mass range over which the ${ }^{14} \mathrm{~N}$-rich region extends amounts only to $\approx 4 \times 10^{-9} M_{\odot}$. Eventually, the ${ }^{14} \mathrm{~N}$-pocket is swept into the helium-flash convection zone during the next pulse.

\subsection{White dwarf evolution}

After considerable mass loss, the mass of the hydrogen envelope is reduced so much that the WD progenitor abandons the thermally pulsing AGB phase and evolves towards large $T_{\text {eff }}$ values to becomes a WD. By the end of mass loss at $T_{\mathrm{eff}} \approx 8000 \mathrm{~K}$, the mass of the hydrogen envelope of the $0.936-M_{\odot}$ remnant (sequence NOV) amounts to $M_{\mathrm{H}} \approx 5.5 \times$ $10^{-5} M_{\odot} .200 \mathrm{yr}$ later, the post-AGB remnant reaches the point of maximum $T_{\text {eff }}$ and $M_{\mathrm{H}}$ is reduced to $10^{-5} M_{\odot}$ as a result of nuclear burning. We mention that a similar value for $M_{\mathrm{H}}$ has also been derived by Blöcker (1995) for his most massive WD remnant. The time-dependence of $M_{\mathrm{H}}$ is displayed in Fig. 7. Notably, by the time the ZZ Ceti domain is reached, subsequent nuclear burning has further reduced the hydrogen mass to $M_{\mathrm{H}} \approx 2.3 \times 10^{-6} M_{\odot}$. This occurs as the WD evolves through the $T_{\text {eff }}\left(\log L / L_{\odot}\right)$ range of $140000-25000 \mathrm{~K}$ (1.65-1.55). Most of this reduction is the result of nuclear burning via $\mathrm{CNO}$ of hydrogen chemically diffusing inwards. For comparison, when diffusion is neglected, the final hydrogen content remains about $5.3 \times 10^{-6} M_{\odot}$ once nuclear burning via $\mathrm{CNO}$ becomes virtually extinct at $T_{\text {eff }} \approx 50000 \mathrm{~K}$ after $1.6 \times 10^{6} \mathrm{yr}$ of evolution. We want to mention that because we have not invoked additional mass loss episodes during the planetary nebula stage or early during the WD cooling branch, the

\footnotetext{
3 A process which is also favoured by the occurrence of overshoot at the bottom of the helium-flash convection zone.
} 


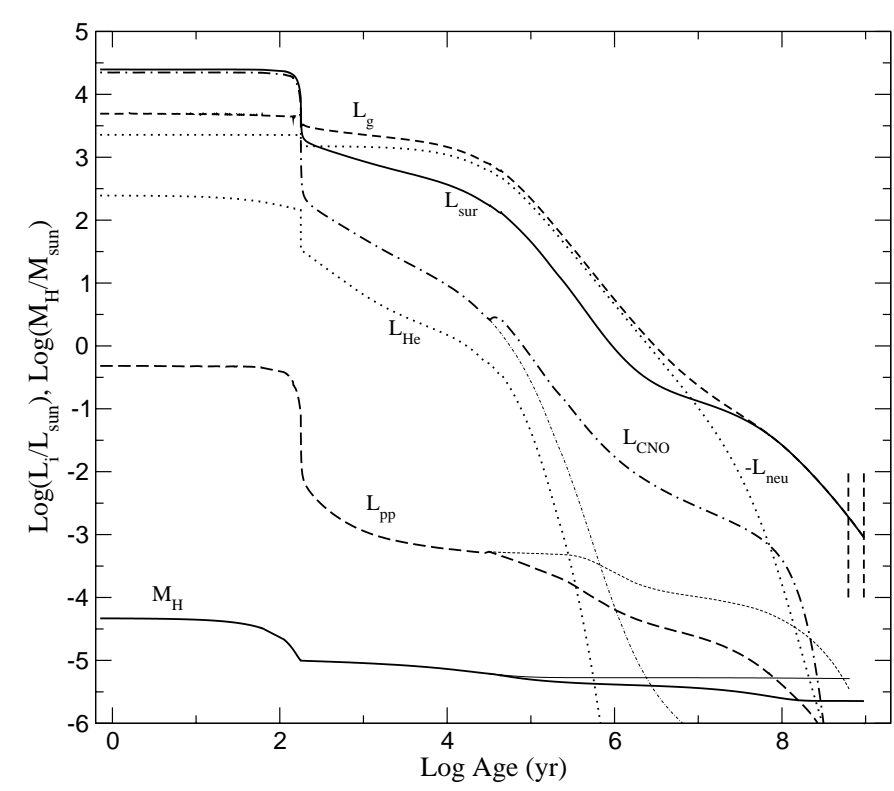

Fig. 7. Time-dependence of different luminosity contributions and mass of hydrogen envelope $\left(M_{\mathrm{H}}\right)$ (in solar units) for the $0.936-M_{\odot}$ WD remnant of 7.5- $M_{\odot}$ evolution (sequence NOV): surface luminosity, $L_{\mathrm{sur}}$, luminosity due to proton-proton reactions, $L_{\mathrm{pp}}$, CNO bicycle, $L_{\mathrm{CNO}}$, helium burning, $L_{\mathrm{He}}$, neutrino losses, $L_{\mathrm{neu}}$ and rate for gravothermal (compressional plus thermal) energy release $L_{\mathrm{g}}$. Thin lines depict $L_{\mathrm{CNO}}, L_{\mathrm{pp}}$ and $M_{\mathrm{H}}$ for the case when element diffusion is neglected. The approximate location of the ZZ Ceti instability strip is indicated with vertical dashed lines. Note that by the time the ZZ Ceti domain is reached, hydrogen burning is virtually extinguished. As a result of hydrogen chemically diffusing inwards, nuclear burning via CNO remains non-negligible at late times and the final hydrogen envelope mass becomes a factor two smaller than when diffusion is not considered. Time is in years counted from the end of mass loss.

value of $M_{\mathrm{H}}$ should be considered as an upper limit. Because of the larger surface gravity characterizing massive WDs, the resulting hydrogen envelope is less massive than in a typically $0.6 M_{\odot} \mathrm{WD}$. For instance, for a $0.563 M_{\odot} \mathrm{WD}$ model, Althaus et al. (2002) find a hydrogen envelope mass of $7 \times 10^{-5} M_{\odot}$.

In Fig. 7 we also show as a function of time the luminosity contributions due to hydrogen burning via proton-proton reactions $L_{\mathrm{pp}}$ and $\mathrm{CNO}$ bi-cycle $L_{\mathrm{CNO}}$, helium burning $L_{\mathrm{He}}$, neutrino losses $L_{\text {neu }}$, surface luminosity $L_{\text {sur }}$ and gravothermal energy release $L_{\mathrm{g}}$ for the $0.936-M_{\odot}$ WD remnant (sequence NOV) from the end of mass loss episodes near the AGB to the domain of the ZZ Ceti stars. In Fig. 7 we also include the predictions for hydrogen burning luminosities and hydrogen envelope mass for the situation when element diffusion is neglected (thin lines). Some features of this figure deserve comment. In particular, except for first $200 \mathrm{yr}$ when the remnant evolves to its WD configuration, nuclear burning never constitutes the main source of surface luminosity of the star. Note that had diffusion not been considered, nuclear burning via $\mathrm{CNO}$ cycle reactions would have ceased after only $10^{6} \mathrm{yr}$ of evolution, a result which is in agreement with that of the Blöcker (1995) calculations. By contrast, $\mathrm{CNO}$ reactions at the base of the hydrogen envelope remain non-negligible for a longer period of time $\left(2 \times 10^{8} \mathrm{yr}\right)$ in the WD evolution if diffusion is allowed to operate. This

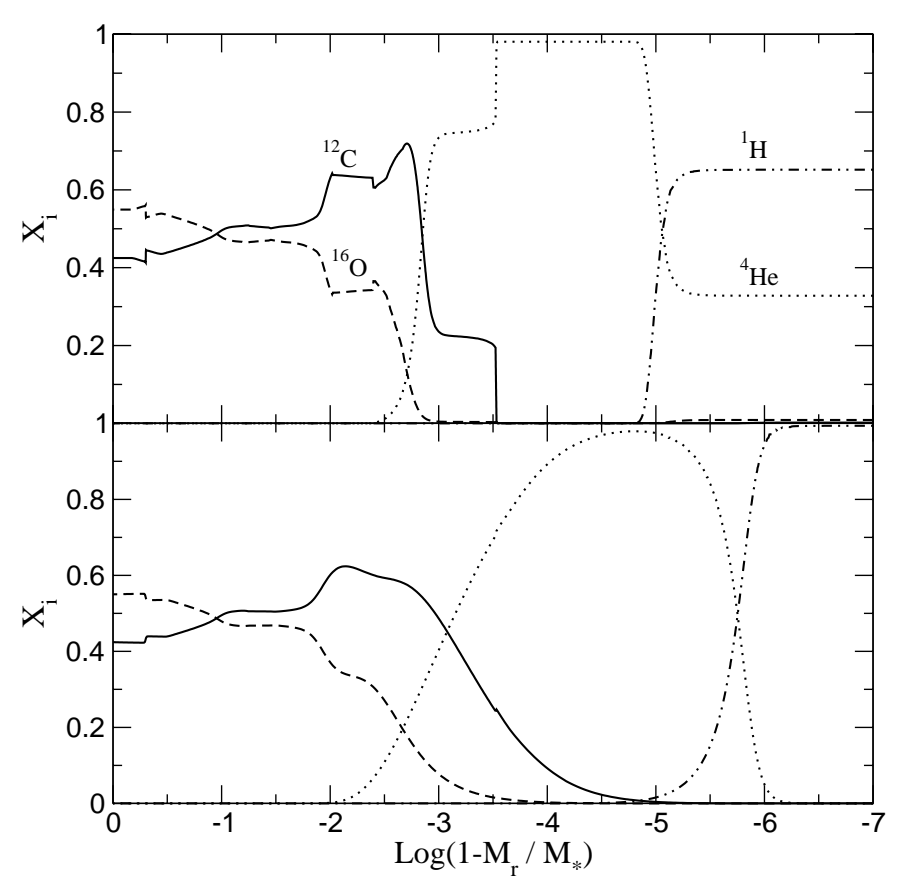

Fig. 8. Abundance by mass of ${ }^{1} \mathrm{H},{ }^{4} \mathrm{He},{ }^{12} \mathrm{C}$ and ${ }^{16} \mathrm{O}$ as a function of the outer mass fraction for the $0.936-M_{\odot}$ WD remnant of 7.5- $M_{\odot}$ evolution (sequence NOV) at the start of the cooling branch (upper panel) and near the ZZ Ceti instability strip (bottom panel). Models are characterized by values $\left(\log \left(L / L_{\odot}\right), \log T_{\text {eff }}\right)$ of $(2.25,5.27)$ and $(-2.92$, 4.06) (upper and bottom panel, respectively). Clearly, element diffusion strongly affects the chemical profile.

is because chemical diffusion carries some hydrogen inwards into the helium buffer and carbon upwards from the carbonrich zone through the buffer layer (see later in this section), thus favouring the occurrence of nuclear reactions. However, by the time the $\mathrm{ZZ} \mathrm{Ceti} \mathrm{domain} \mathrm{is} \mathrm{reached,} \mathrm{hydrogen} \mathrm{burning}$ becomes virtually extinct.

From the preceding section, we saw that the chemical stratification for both the core and outer layers of the WD remnant is quite different according to whether overshooting is considered or not during the pre-WD evolution. We show the chemical abundance distribution at the start of the WD cooling branch in the upper panel of Figs. 8 and 9 for the 0.936- and 0.94- $M_{\odot}$ WD remnants (sequence NOV and OV), respectively. The external chemical profile emerging from the thermally pulsing AGB phase (Sect. 3.1) is characterized by a region rich both in helium and carbon (and oxygen if overshooting is considered), the relics of the short-lived mixing episode during the peak of the last helium pulse on the AGB, and an overlying helium-rich buffer. The mass of the helium buffer amounts to $3 \times 10^{-4}$ and $9.4 \times 10^{-4} M_{\odot}$ for sequences NOV and OV, respectively. The buffer in sequence NOV is substantially less massive than in sequence OV simply because the WD progenitor in sequence NOV departs from the AGB at an early stage during the interpulse phase, whilst the initial chemical stratification for the WD in sequence OV corresponds to a pre-WD structure at an evolutionary stage well advanced in the interpulse phase. Note also the signatures left by the third and last dredge-up episode during the last helium thermal pulse in sequence OV, in which 


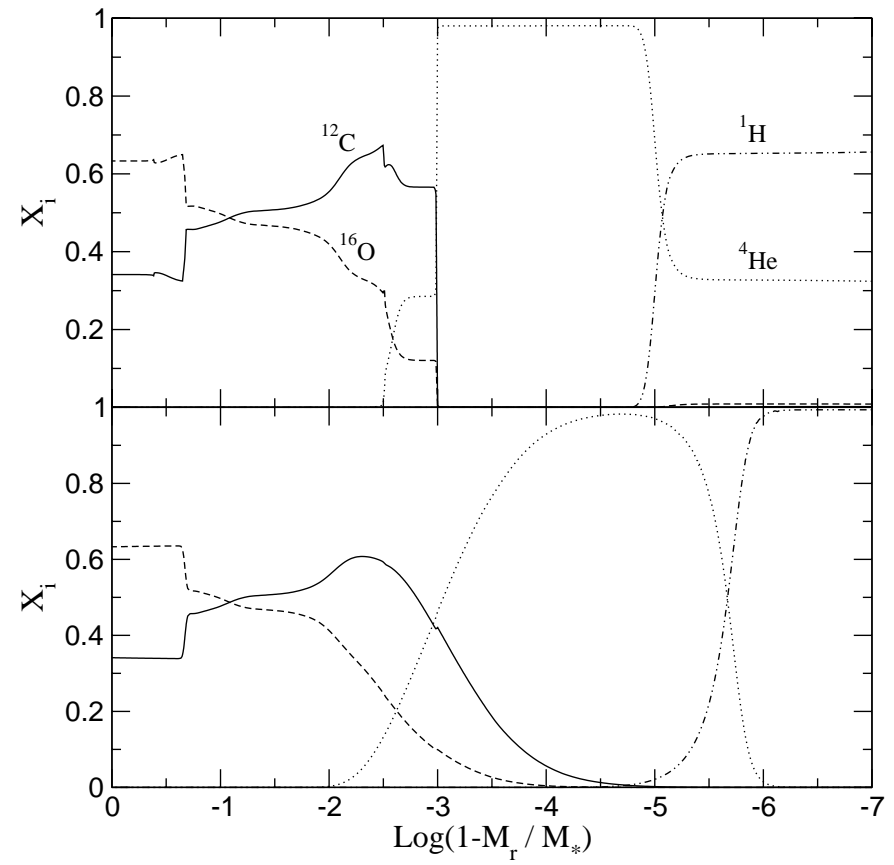

Fig. 9. Same as figure 8 but for the $0.94-M_{\odot}$ WD remnant of $6-M_{\odot}$ evolution (sequence OV). Note that, except for the inner region of the core, element diffusion acting during the WD evolution is so efficient that the resulting abundance distribution at the ZZ Ceti stage does not depend on whether overshooting during the thermally pulsing AGB phase is considered or not.

convection penetrated into the carbon-rich region (see Fig. 6 for details). It is also clear from these figures the markedly different chemical profile resulting from the consideration of core overshooting during central helium burning.

On the cooling track, the abundance distribution of the WD remnant will be strongly modified by gravitational settling and chemical diffusion. This is illustrated in the bottom panel of Figs. 8 and 9 which show the chemical profiles at the ZZ Ceti stage for sequence NOV and OV. These figures emphasize the role of element diffusion in the external chemical stratification of massive ZZ Ceti stars. Indeed, the shape of the chemical profile that was built-up during the AGB phase is virtually wiped out by diffusion processes acting during WD evolution. In particular, near- discontinuities left by past mixing episodes in the shape of the external chemical interfaces (of primary importance in pulsation properties of WDs) are strongly smoothed out by the time the WD has approached the hot edge of the ZZ Ceti domain. It is worth noting that, except for the inner part of the core, element diffusion is so efficient that the resulting abundance distribution at the $\mathrm{ZZ}$ Ceti stage does not depend on whether overshooting during the thermally pulsing AGB phase is considered or not. In fact, the external chemical profile at the $\mathrm{ZZ}$ Ceti stage is quite similar for both sequences. By contrast, towards the innermost region, diffusion time scale becomes much longer than the evolutionary time scale, and the chemical profile remains therefore fixed during the whole WD evolution ${ }^{4}$. Thus, overshoot episodes occurring during the

\footnotetext{
${ }^{4}$ Except for the minor mixing episode at the innermost ${ }^{12} \mathrm{C} /{ }^{16} \mathrm{O}$ core induced by an inward-decreasing molecular weight.
}

pre-WD evolution leave recognizable features in the chemical profile of massive ZZ Ceti stars solely at the inner region of the core, features that are expected to leave their imprints on the theoretical period spectrum of these variable WDs (see Sect. 4). As mentioned, our model is characterized by a chemical interface in which helium, carbon and oxygen in non-negligible abundances coexist, an interface which, at the ZZ Ceti stage, has extended appreciably as a result of chemical diffusion.

The evolution of the chemical abundance distribution caused by diffusion processes can best be seen in Fig. 10, which illustrates the abundances by mass of ${ }^{1} \mathrm{H},{ }^{3} \mathrm{He},{ }^{4} \mathrm{He},{ }^{12} \mathrm{C},{ }^{14} \mathrm{~N}$ and ${ }^{16} \mathrm{O}$ for the $0.936-M_{\odot}$ WD remnant (sequence NOV) as a function of the outer mass fraction at various epochs characterized by values of $\log L / L_{\odot}$ and $\log T_{\text {eff }}$ (numbers given in parentheses). Panel a shows the chemical stratification at the start of the cooling branch at high luminosities. In the outermost layers, chemical composition corresponds basically to that fixed by the second dredge-up episode during the early AGB phase. In the helium buffer, CNO abundances are different from those in the outer layers, because hydrogen burning in earlier evolutionary phases processed almost all the initial ${ }^{12} \mathrm{C}$ and ${ }^{16} \mathrm{O}$ into ${ }^{14} \mathrm{~N}$. Rapidly, gravitational settling and chemical diffusion begin to play an increasing role. Indeed, gravitational settling causes hydrogen to float to the surface and heavier elements to sink down to such an extent that $4.2 \times 10^{5} \mathrm{yr}$ later and in the absence of any competing process, there are no metals in the outermost $10^{-6} M_{\odot}$ of the star (see panel b). With subsequent cooling, the effect of chemical diffusion is clearly seen at the chemical interfaces where large abundance gradients exist. As a result, in the helium buffer there is a tail of hydrogen reaching hotter layers and a tail of carbon diffusing outwards from the carbon-rich zone (panel c), thus causing hydrogen burning via CNO cycle to be efficient for a longer period of time than when diffusion is neglected (see Fig. 7). Notably, the tail of the hydrogen distribution reaches a maximum depth by this epoch. Note also that by this time, diffusion has completely altered the shape of the chemical profile below the helium buffer. $7.3 \times 10^{8}$ yr later, the star reaches the $\mathrm{ZZ} \mathrm{Ceti}$ domain (panel d). During this time, the tail of the carbon distribution has diffused outwards until reaching the outer boundary of the helium buffer. Also, the tail of hydrogen begins to retreat outwards as a result of gravitational settling. This is so because electron degeneracy causes chemical diffusion to become less important and the inward diffusion of hydrogen is stopped. It is interesting to see that, despite the fact that the diffusion of hydrogen and carbon into the helium buffer zone has led to sufficiently large abundances of these two elements by the time the ZZ Ceti stage is reached, the temperature at the base of the hydrogen envelope is too low $\left(\approx 5 \times 10^{6} \mathrm{~K}\right)$ for hydrogen burning to be of any importance. A last remark regarding the influence of diffusion is related to the evolution of ${ }^{12} \mathrm{C}$ and ${ }^{14} \mathrm{~N}$ abundances in the buffer layer. Indeed, initially the ${ }^{14} \mathrm{~N}$ abundance in the helium buffer overwhelms that of ${ }^{12} \mathrm{C}$ (see panel a), but before the $\mathrm{ZZ}$ Ceti stage is reached, ${ }^{14} \mathrm{~N}$ is less abundant than ${ }^{12} \mathrm{C}$ throughout the star despite nuclear burning having processed considerable ${ }^{12} \mathrm{C}$ into ${ }^{14} \mathrm{~N}$ along WD evolution. The same finding, though to a lesser extent, has been reported by Iben \& MacDonald (1986) for the case of a 0.6 $M_{\odot}$ WD. 

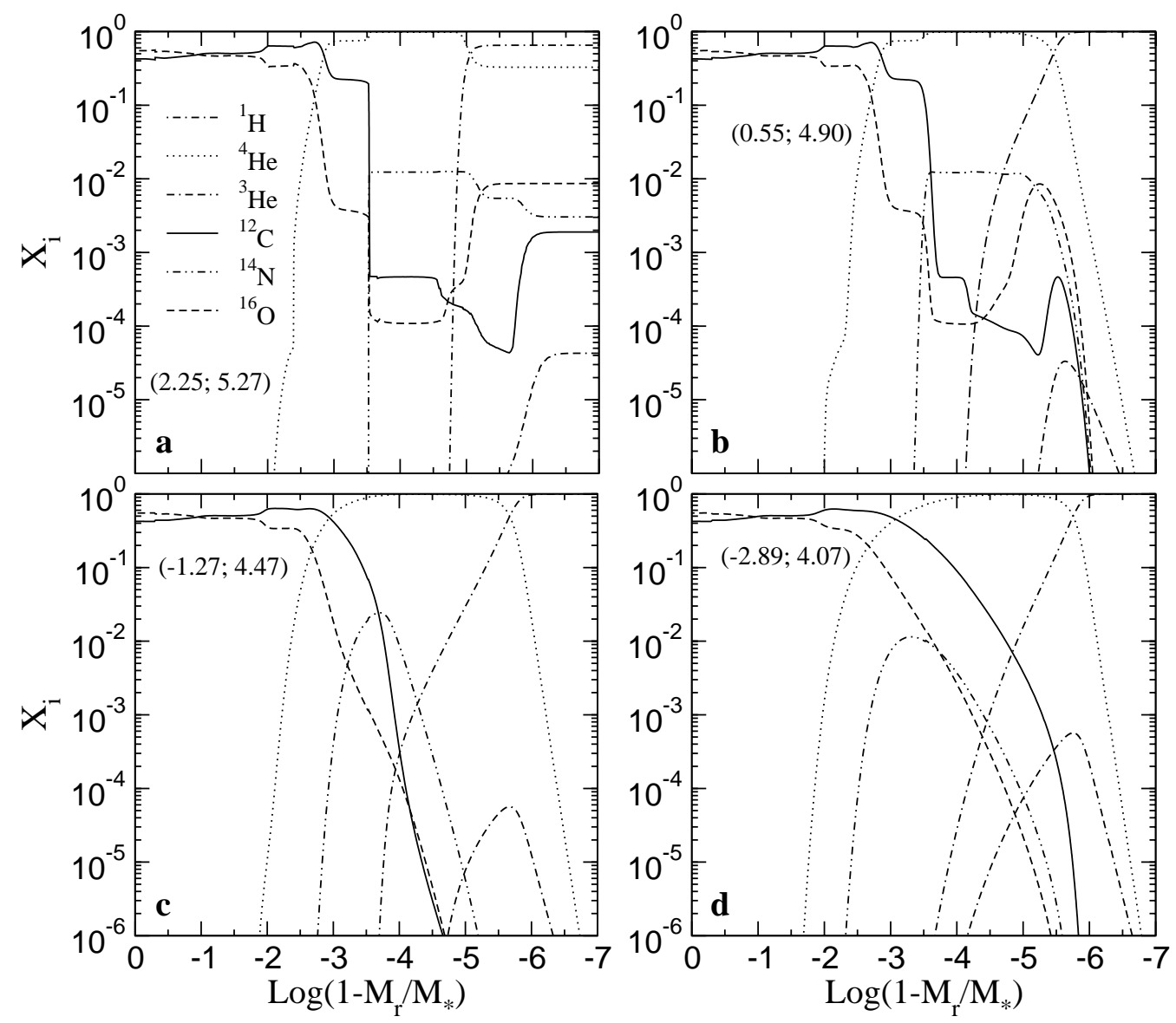

Fig. 10. Abundance by mass of ${ }^{1} \mathrm{H},{ }^{3} \mathrm{He},{ }^{4} \mathrm{He},{ }^{12} \mathrm{C},{ }^{14} \mathrm{~N}$ and ${ }^{16} \mathrm{O}$ in terms of the outer mass fraction for the $0.936-M_{\odot}$ WD remnant of 7.5- $M_{\odot}$ evolution (sequence NOV) at four evolutionary stages characterized by values of $\log L / L_{\odot}$ and $\log T_{\text {eff }}$ (numbers given between brackets). Panel a) corresponds to the start of the cooling branch, and panel d) to the ZZ Ceti domain. At this stage, note the inward (outward) extent of the tail of the hydrogen (carbon) distribution resulting from chemical diffusion.

In what follows we explore the implications for the global pulsational properties of our models.

\section{Pulsational results}

For the pulsational analysis we have chosen two template ZZ Ceti models with $T_{\text {eff }} \approx 12000 \mathrm{~K}$, corresponding to sequences NOV and OV. In addition, we compare our results with the pulsational predictions for a $0.94-M_{\odot}, T_{\text {eff }}=12000 \mathrm{~K}$ WD model, as given by the stellar modeling considered in Montgomery \& Winget (1999) (hereafter MW model). We want to mention that despite the fact that the models are partially crystallized (between $\sim 8 \%$ at the blue edge, to $\sim 25 \%$ at the red edge of the instability strip), we have computed the theoretical period spectrum assuming that the model interior is in a completely uncrystallized, fluid state. A complete discussion of the pulsational properties taking crystallization selfconsistently into account would be quite lengthy, so we have decided to postpone this to an upcoming communication, for which we will compute more massive WD models (and thus more suitable for the study of crystallized WDs) than attempted here.

\subsection{The Ledoux term and the Brunt-Väisälä frequency}

The Brunt-Väisälä frequency $(N)$, a fundamental quantity in WD pulsations, is computed by employing the "modified Ledoux" prescription. Specifically, $N$ is given by (see Brassard et al. 1991 for details)

$N^{2}=\frac{g^{2} \rho}{P} \frac{\chi_{\mathrm{T}}}{\chi_{\rho}}\left(\nabla_{\mathrm{ad}}-\nabla+B\right)$.

The Ledoux term $B$, for the case of a multicomponent plasma (M-component plasma), is given by

$B=-\frac{1}{\chi_{\mathrm{T}}} \sum_{i=1}^{M-1} \chi_{X_{i}} \frac{\mathrm{d} \ln X_{i}}{\mathrm{~d} \ln P}$

Here, $\chi_{\mathrm{T}}\left(\chi_{\rho}\right)$ denotes the partial logarithmic pressure derivative with respect to $T(\rho), \nabla$ and $\nabla_{\text {ad }}$ are the actual and adiabatic temperature gradients, respectively, $X_{i}$ is the abundance by mass of species $i$, and

$\chi_{x_{i}}=\left(\frac{\partial \ln P}{\partial \ln X_{i}}\right)_{\rho, T,\left\{X_{j \neq i}\right\}}$,

where $P$ is the pressure. It is worth mentioning that the Ledoux term accounts explicitly for the contribution to $N$ from any 


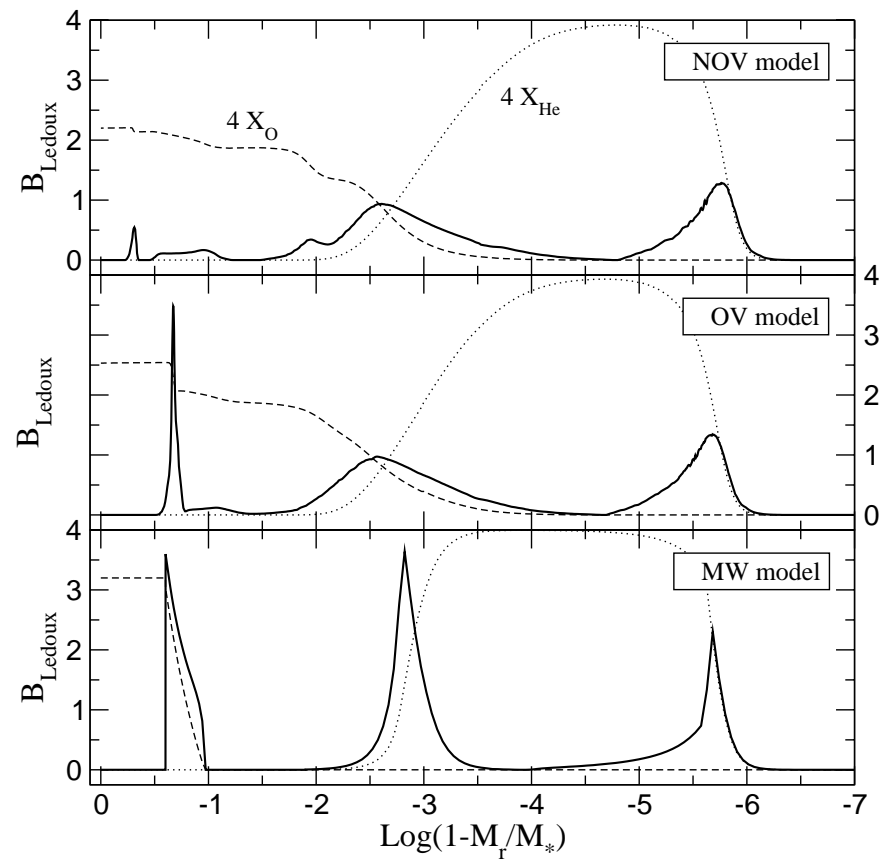

Fig. 11. The Ledoux term $B$ in terms of the outer mass fraction as predicted by the NOV, OV and MW models (upper, middle and bottom panel). In order to make easier the identification of the various features exhibited by $B$, the mass fraction of ${ }^{16} \mathrm{O}$ (dashed lines) and ${ }^{4} \mathrm{He}$ (dotted lines) is depicted, with their magnitudes arbitrarily increased by a factor of 4 for clarity.

change in composition in the model (the zones of chemical transition). Brassard et al. (1992a) stress the relevance of a correct treatment of $N$ at the interfaces of chemical composition transition zones in stratified WDs, particularly in connection with the phenomenon of mode trapping.

In Fig. 11, the Ledoux term is plotted as a function of the outer mass fraction for NOV, OV and MW models (upper, middle and bottom panel, respectively). In addition, we have plotted the abundance by mass of ${ }^{16} \mathrm{O}$ (dashed lines) and ${ }^{4} \mathrm{He}$ (dotted lines) scaled by a factor 4 for a clear and easy visual interpretation of the different features exhibited by $B$ (see from Eq. (19) the strong dependence of $B$ on the slopes of the chemical abundances). To begin with, note that the Ledoux term in models NOV and OV shows smoother peaks in the outer layers, as compared with the case of MW model. This different behaviour can be in part understood on the basis that MW invoke the diffusive equilibrium in the trace element approximation (see Tassoul et al. 1990) to calculate the chemical profile at the outer chemical interfaces, an approximation that leads to pronounced peaks in the $B$ term. Note that the $B$ term for OV model is characterized by a high-amplitude peak towards the innermost regions where the presence of overshooting has led to a sharp variation of the ${ }^{12} \mathrm{C} /{ }^{16} \mathrm{O}$ profile (see Figs. 3 and 9). This behaviour is in sharp contrast with the situation expected from the case in which core overshooting is neglected (upper panel of Fig. 11). Note that the MW model also shows a peak in the core. However, at variance with MW, models NOV and OV show an extended bump at the bottom of the helium-rich

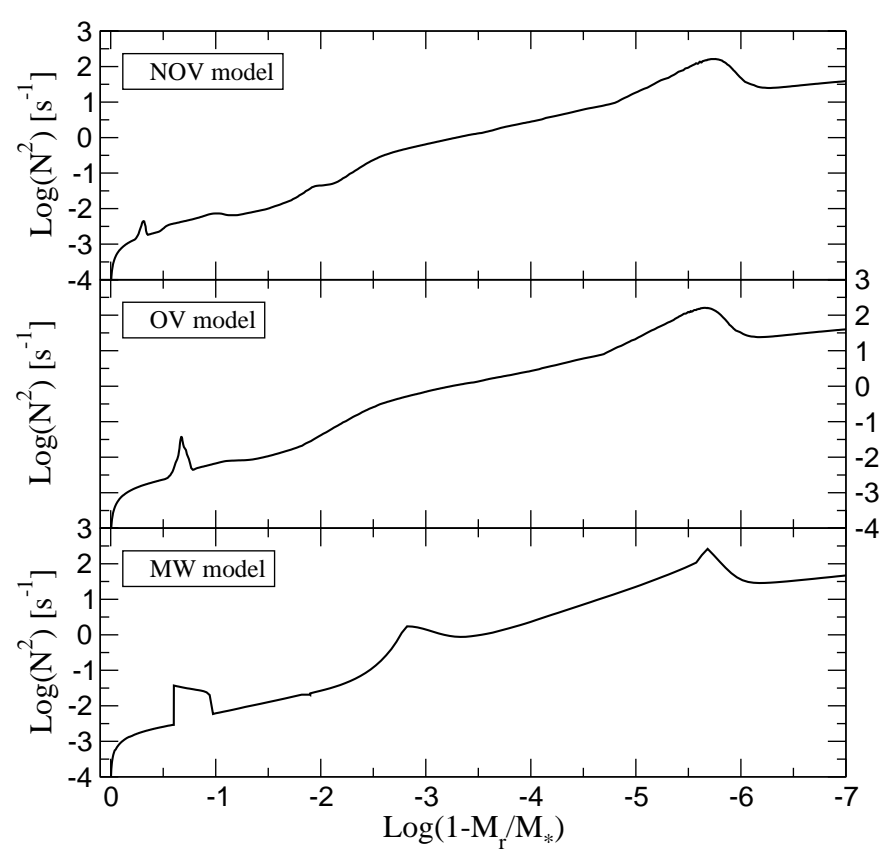

Fig. 12. Same as Fig. 11, but for the logarithm of the squared Brunt-Väisälä frequency.

zone, which again is a result of the broadness and smoothness of the diffusion-modeled profiles.

In Fig. 12 we depict the logarithm of the squared BruntVäisälä frequency for the same cases shown in Fig. 11. Each feature exhibited by the Ledoux term is clearly translated into $N^{2}$. Except for the feature induced by the internal oxygencarbon distribution, the Brunt-Väisälä frequency as predicted by the OV and NOV models is very smooth. In particular, note that the chemical transition regions lead to smooth bumps at $\log q \sim-2.5$ and -5.5 . In contrast, the Brunt-Väisälä frequency corresponding to the MW model shows enhanced peaks at the location of all the chemical interfaces (see bottom panel of Fig. 12).

\subsection{Pulsational properties of the template models}

For the pulsation analysis of the NOV and OV models we have employed the same pulsational code as in Córsico et al. (2001, 2002). We refer the reader to those papers and references therein for details. The boundary conditions at the stellar centre and surface are those given by Osaki \& Hansen (1973) (see Unno et al. 1989 for details). Pulsation computations for the MW model were carried out by employing the same pulsational code as in Montgomery \& Winget (1999) $)^{5}$. For each computed mode we obtain the eigenperiod $P_{k}$ (being $k$ the radial overtone of mode) and the dimensionless eigenfunctions $y_{1}, \ldots, y_{4}$ (see Unno et al. 1989 for their definition). Following previous studies of WD pulsations, the normalization condition adopted is $y_{1}=1$ at the stellar surface. We also compute the oscillation kinetic energy ( $E_{\text {kin }}$; see Eq. (1) of Córsico et al. 2002) and the weight function, $w f$, given by Kawaler et al. (1985).

5 This code employes the outer boundary conditions as in Saio \& Cox (1980). 


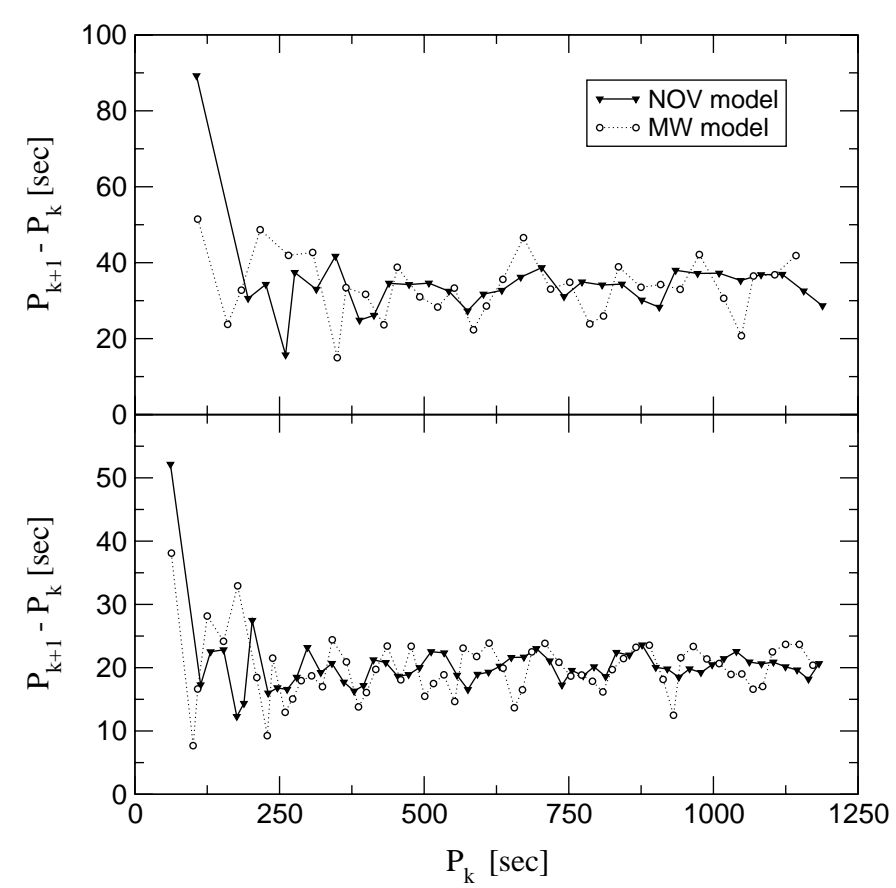

Fig. 13. Period spacing values for $\ell=1$ (upper panel) and 2 (bottom panel) in terms of the computed periods, $P_{k}$ for the NOV model and MW predictions. Filled triangles and solid lines (empty dots and dotted lines) correspond to the NOV (MW) model.

The weight function gives the relative contribution of the different regions in the star to the period formation (see for details Kawaler et al. 1985 and Brassard et al. 1992a,b). Finally, for each model computed we derive the asymptotic spacing of periods as in Tassoul et al. (1990).

For our template models we have computed adiabatic gmodes with $\ell=1,2$ and 3 , with periods in the range expected for ZZ Ceti stars ( $50 \mathrm{~s} \lesssim P_{k} \lesssim 1300 \mathrm{~s}$ ). We begin by examining Fig. 13 which shows the values for the forward period spacing $\Delta P_{k}\left(\equiv P_{k+1}-P_{k}\right)$ for $\ell=1$ (upper panel) and $\ell=2$ (lower panel) in terms of the periods computed. An inspection of the figure reveals that the amplitude of $\Delta P_{k}$ corresponding to the NOV model are typically lower as compared with the results of the MW model. This difference is understood on the basis that the Brunt-Väisälä frequency of the NOV model is smoother than that of the MW model (compare upper and bottom panel of Fig. 12). The marked smoothness of the $\Delta P_{k}$ distribution in the NOV model implies that mode trapping is appreciably diminished in this model.

In Fig. 14 we show the same $\Delta P_{k}-P_{k}$ diagram as in Fig. 13, but this time we compare the results of the OV model with the MW one. At first glance, the $\Delta P_{k}$ values for both set of computations exhibit similar amplitudes. However, a closer inspection of the plot reveals an interesting feature. In fact, the $\Delta P_{k}$ distribution corresponding to the OV model clearly shows periodic minima with values between minima almost constant and tending to the asymptotic value (not plotted). As we shall see towards the end of this section, the minima in $\Delta P_{k}$ correspond to modes partially confined to the deepest regions of the $\mathrm{OV}$ model as a result of the pronounced $N^{2}$-peak showed by middle panel of Fig. 12. By contrast, the $\Delta P_{k}$ distribution for the

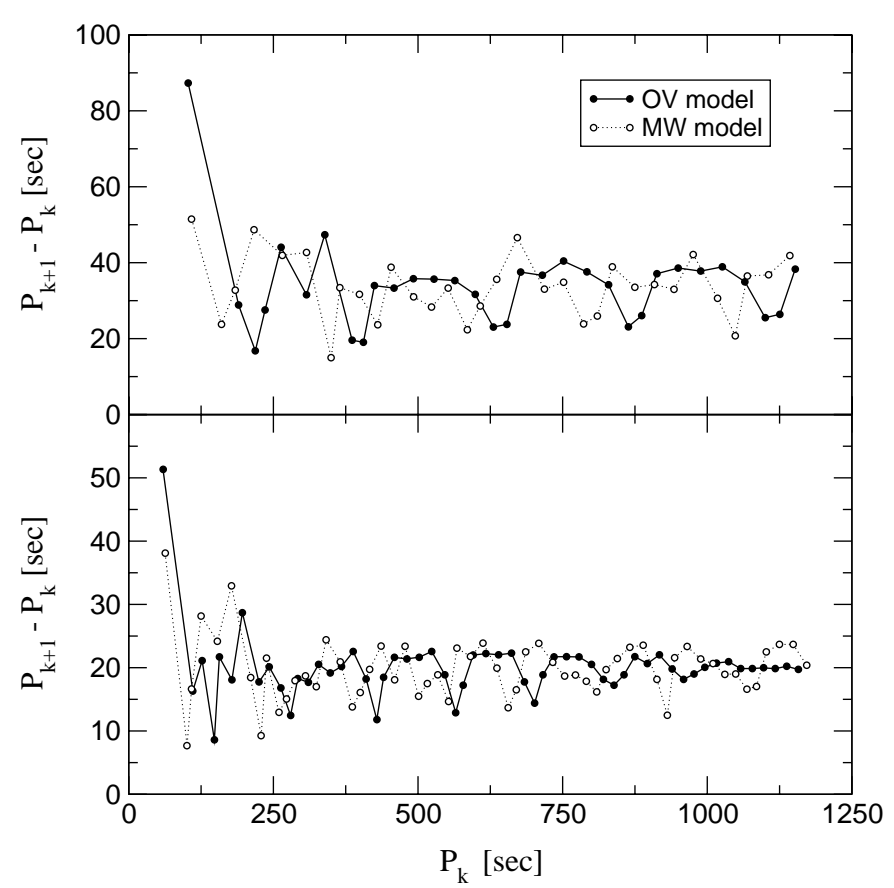

Fig. 14. Same as Fig. 13, but for OV and MW models. Filled dots and solid lines (empty dots and dotted lines) correspond to the OV (MW) model.

MW model shows clear signals of mode trapping with different amplitudes caused by the mode trapping due to the presence of all chemical interfaces in this model.

Finally, in Fig. 15 we compare the $\Delta P_{k}$ distribution corresponding to the NOV and OV models. We can clearly observe that the non-uniformity in the period spacing is much more apparent in the OV model, as expected from the shape of $N^{2}$ corresponding to these cases (Fig. 12). As stated before, the minima in the $\Delta P_{k}$ distribution for the OV model are associated with modes partially confined to the high-density core region placed between the centre of the model and $\log q \sim-0.7$. Consequently, these modes are energy-enhanced, so they must exhibit maxima in the $E_{\text {kin }}$ distribution. To demonstrate this, we show in Fig. 16 the oscillation kinetic energy distribution corresponding to the OV WD model, where we have labeled in particular the $\ell=2$ modes with overtones $k=17,19$ and 21 . Note from Fig. 15 that the $\ell=2, k=19$ eigenmode corresponds to a minimum of $\Delta P_{k}$, but it has actually a $E_{\text {kin }}$ value near a local maximum (Fig. 16).

To convince the reader that minima in $\Delta P_{k}$ correspond to energy-enhanced modes, we depict in Fig. 17 the amplitude of eigenfunctions $y_{1}$ and $y_{2}$ (corresponding to radial and tangential displacements of matter, respectively) for $\ell=2, k=17,19$ and 21 modes at the central region of the OV model. In addition, we show in the bottom panel the weight function corresponding to these modes. An inspection of this figure reveals that the amplitude of the eigenfunctions as well as the weight function for the $k=19$ mode are noticeably larger as compared with the neighboring ones $(k=17,21)$. Note also the evident distortion of the shape in $y_{2}$ and $w f$ for the modes at the location of the acute peak in the Ledoux term $(\log q \sim-0.7$; middle panel of Fig. 10). This feature, which is a direct consequence 


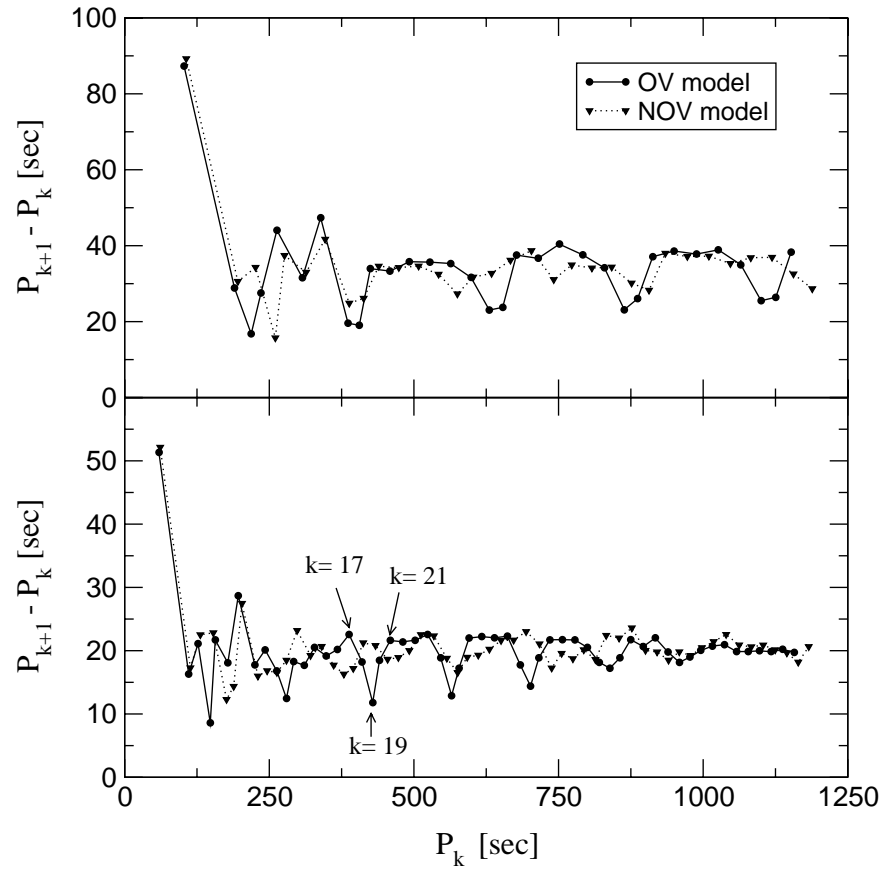

Fig. 15. Same as Fig. 12, but for OV and NOV models. Filled dots (triangles) correspond to the $\mathrm{OV}$ (NOV) model.

of the abrupt fall of $X_{16} \mathrm{O}$, acts to enlarge the magnitude of $y_{1}$, $y_{2}$ and $w f$ of the $k=19$ mode, but diminishes the amplitude of these functions in the case of modes with $k=17$ and 21 .

\subsection{Beyond the trace element approximation}

The trace element approximation has been a workhorse of chemical profile calculations for many years. Sadly, as these and other calculations have shown, it provides an inaccurate description of the transition zone and its associated Ledoux term: it is too narrow, too peaked, and its first derivative is not continuous. In this section, we present a new prescription which retains the simplicity of the trace element approximation but which addresses its problems.

From timescale arguments (e.g., Michaud \& Fontaine 1979) and numerical experiments, we expect the ${ }^{4} \mathrm{He} / \mathrm{H}$ transition zone to be near diffusive equilibrium, so we are justified in using Eq. (A.5) of Arcoragi \& Fontaine (1980) for the equilibrium profiles. Traditionally, the trace approximation results from solving this equation in the limit in which one of the two species is considered to be a trace. However, assuming complete ionization of both species, we find that Eq. (A.5) may still be integrated in closed form. For the ${ }^{4} \mathrm{He} / \mathrm{H}$ zone, we obtain

$C \frac{P}{P_{0}}=\gamma^{\frac{1}{5}}(1+\gamma)^{\frac{18}{25}}(1+2 \gamma)^{-\frac{1}{10}}(1+6 \gamma)^{-\frac{1}{50}}$,

where $\gamma=n_{\mathrm{He}} / n_{\mathrm{H}}$ is the ratio of number densities of the two species, $P$ is the pressure, $P_{0}$ is some reference pressure, and $C$ is a constant. Additionally, if we assume a geometrically thin hydrogen layer (which is certainly valid), then the pressure can be directly related to the mass depth, so that this equation becomes

$\tilde{C} \frac{q_{s}}{q_{\mathrm{H}}}=\gamma^{\frac{1}{5}}(1+\gamma)^{\frac{18}{25}}(1+2 \gamma)^{-\frac{1}{10}}(1+6 \gamma)^{-\frac{1}{50}}$,

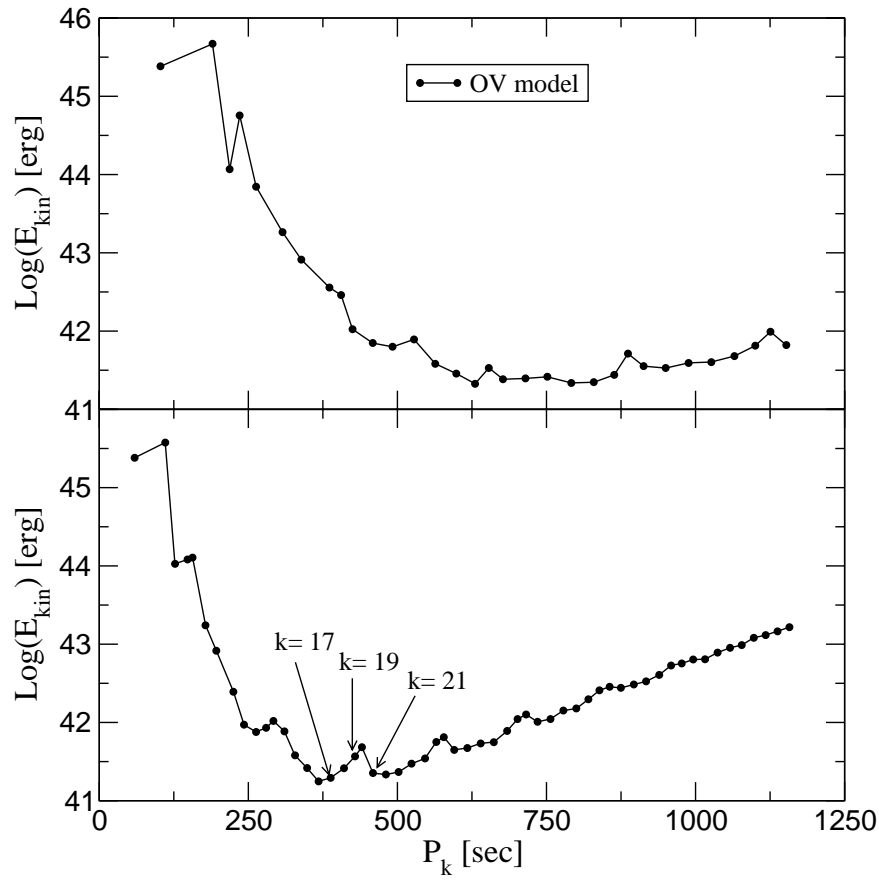

Fig. 16. Kinetic energy values for $\ell=1$ and 2 in terms of the computed periods, $P_{k}$ for the OV model.

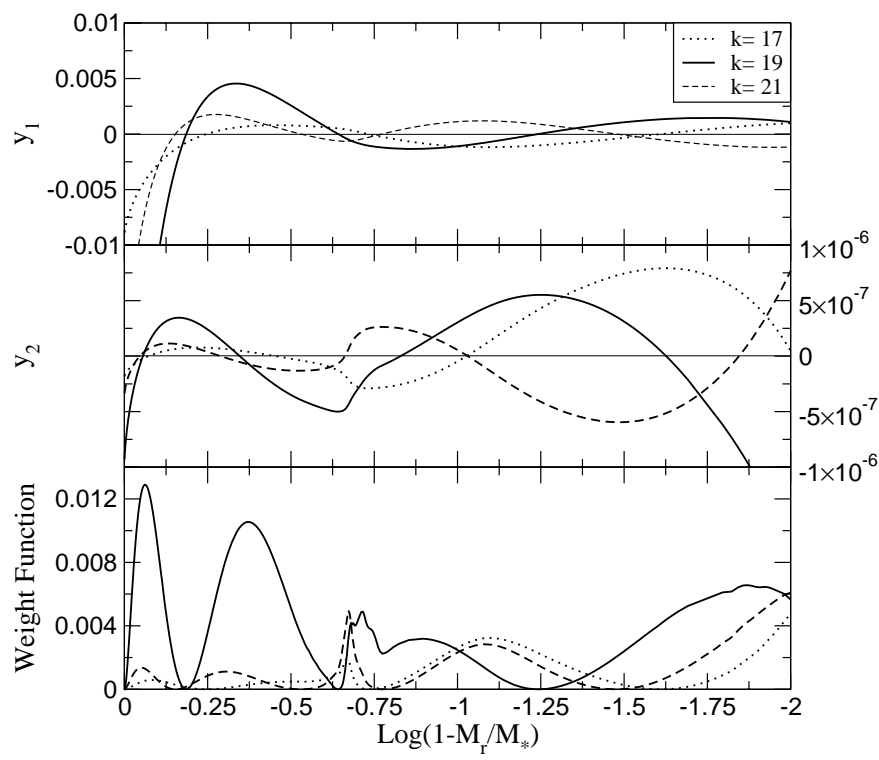

Fig. 17. Eigenfunctions $y_{1}, y_{2}$ and the weight function $w f$ (upper, middle and bottom panel, respectively) for modes with $\ell=2$ and $k=17$ (dotted lines), $k=19$ (solid lines) and $k=21$ (dashed lines). See text for details.

where $q_{s}=1-M_{r} / M_{*}, q_{\mathrm{H}}=1-M_{\mathrm{H}} / M_{*}$, and $\tilde{C} \approx 1.764$. Since the mass fraction of He is related to $\gamma$ by

$X_{\mathrm{He}}=\frac{A_{\mathrm{He}} n_{\mathrm{He}}}{A_{\mathrm{H}} n_{\mathrm{H}}+A_{\mathrm{He}} n_{\mathrm{He}}}=\frac{4 \gamma}{1+4 \gamma}$,

Eq. (22) gives us a smooth relation for the mass fraction of $\mathrm{He}$ valid from large abundances $\left(X_{\mathrm{He}} \sim 1, \gamma \gg 1\right)$ to low abundances $\left(X_{\mathrm{He}} \ll 1, \gamma \ll 1\right)$.

Since the treatment given above offers an easy implementation of diffusion profiles, we would like to see how it compares 
with self-consistently computed profiles. In Fig. 18 we compare the profile calculated using Eq. (22) with that obtained from an evolutionary calculation assuming self-consistent, time-dependent diffusion. We see that our parameterized profile provides a good representation of the overall profile shape, and that it reproduces the maximum height of the Ledoux term to within $\sim 20 \%$. Since the numerical implementation of this prescription is only slightly more expensive computationally than that based on the trace element approximation, we recommend Eq. (22) as a reasonable replacement in codes which use a pre-specified functional form for the profile shapes.

The main discrepancy between the two profiles shown in Fig. 18 is most likely due to the neglect of electron degeneracy pressure which is implicit in the use of Eq. (A.5) of Arcoragi \& Fontaine (1980). Since the deeper C-O/He transition zone is in a more degenerate environment, as well as being much farther away from diffusive equilibrium, a derivation along the lines which led to Eq. (22) is more difficult to justify; we therefore defer a discussion of a simple functional form for its shape until a future paper. Finally, we note that the above prescription for the ${ }^{4} \mathrm{He} / \mathrm{H}$ profile is aimed mainly at calculating the BruntVäisälä frequency. As such, it may need to be truncated at large depths in order to prevent unphysical situations such as a thermonuclear runaway due to $\mathrm{H}$ burning.

\section{Conclusions}

In this work we have computed new and improved evolutionary models for carbon-oxygen DA WD stars appropriate for the study of massive ZZ Ceti stars. In addition, the implications of our new models for the pulsational properties of massive ZZ Ceti stars have been explored. To this end, we have followed the complete evolution of massive WD progenitors from the zero-age main sequence through the thermally pulsing and mass loss phases on the AGB to the WD regime. Attention has been focused on the modeling of the chemical abundance distribution. In this regard, we developed a timedependent scheme for the simultaneous treatment of chemical changes caused by nuclear burning and mixing processes. Salt finger mixing, semiconvection and diffusive overshooting above and below any formally convective zone have been fully accounted for during the pre-WD regime. In this work, we have taken into account an extended mixing length theory for fluids with composition gradients. Also, time-dependent element diffusion for multicomponent gases has been considered during the WD evolution.

An important aspect of the study has been to explore the implications of the occurrence of overshooting during the preWD evolution for the pulsational properties of massive ZZ Ceti stars. To this end, we restrict ourselves to examining two cases of evolution for the progenitor: sequence NOV based on the evolution of a 7.5- $M_{\odot}$ initial mass star in which overshooting was not considered and sequence OV based on the evolution of a 6- $M_{\odot}$ star with overshooting. For both sequences, the mass of the resulting ${ }^{12} \mathrm{C} /{ }^{16} \mathrm{O}$ core is quite similar $\left(\approx 0.94 M_{\odot}\right)$. This has allowed us in principle to compare the pulsational properties of carbon-oxygen massive ZZ Ceti stars having the same stellar
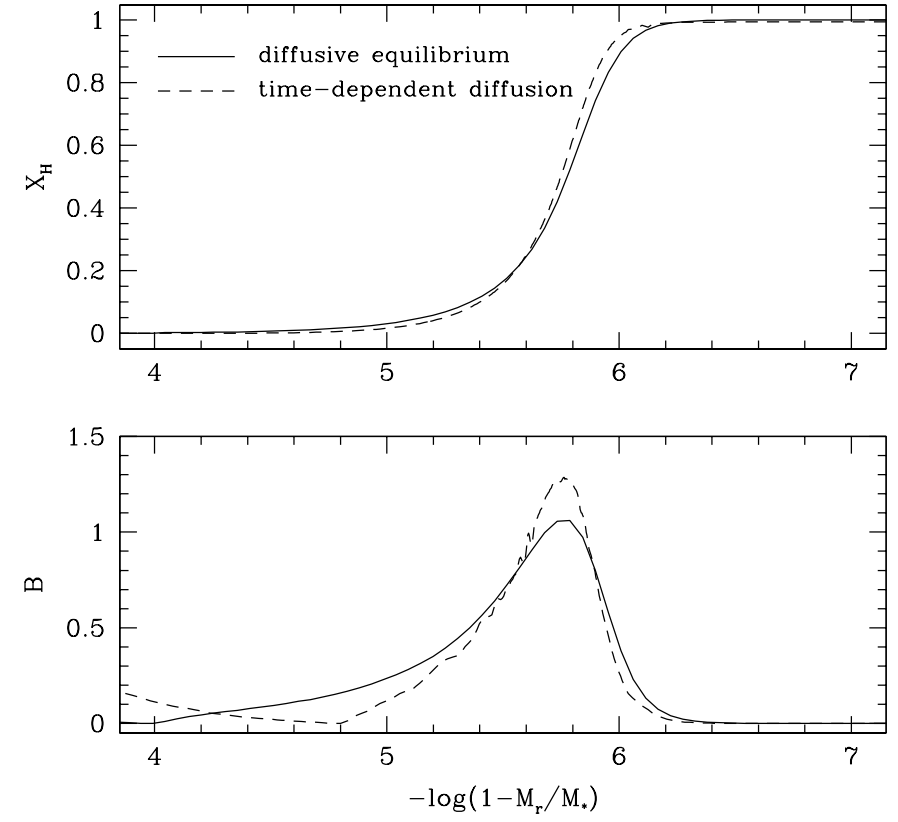

Fig. 18. A comparison of the hydrogen mass fraction (upper panel) and the Ledoux $B$ term (lower panel) for the profile computed using Eq. (22) (solid line), which assumes diffusive equilibrium, complete ionization, and an ideal gas equation of state, with that assuming full time-dependent diffusion (dotted line).

mass but being the result of the evolution of progenitor stars with markedly different initial masses.

As for the main evolutionary results for the WD progenitor, we mention:

- Both of the sequences experience the second dredge-up episode during which the surface composition is appreciably modified from the assumed interstellar abundances.

- A salt finger instability develops after the end of central helium burning, which is particularly noticeable in the case without core overshooting. This instability is responsible for the redistribution of the innermost ${ }^{12} \mathrm{C} /{ }^{16} \mathrm{O}$ profile occurring before the end of the early AGB phase.

- Sequence OV exhibits a sharp variation of the ${ }^{12} \mathrm{C} /{ }^{16} \mathrm{O}$ composition around $M_{r} \approx 0.72 M_{\odot}$, variation which leaves its signature on the theoretical period spectrum (see below).

- During the thermally pulsing phase, the temperature at the base of the convective envelope becomes high enough for hot bottom burning to occur. This is particularly noticeable for sequence $\mathrm{OV}$.

- The presence of overshooting below the bottom of the helium-flash convection zone gives rise to an enhancement of oxygen and carbon in the intershell region below the helium buffer, as compared with models without overshoot. This is in agreement with Herwig (2000) for the case of low- and intermediate-mass stars. 
- Sequence OV experiences the third dredge-up. At the end of this episode, a ${ }^{14} \mathrm{~N}$-pocket is built-up at the base of the helium buffer.

For the WD evolution, our main conclusions are:

- The mass of the hydrogen envelope left at the ZZ Ceti domain amounts to $M_{\mathrm{H}} \approx 2.3 \times 10^{-6} M_{\odot}$. This is about half as large as for the case when element diffusion is neglected.

- As a result of chemical diffusion, CNO reactions remain non-negligible for a considerable period of time $\left(2 \times 10^{8} \mathrm{yr}\right)$ during the WD evolution. However, by the time the ZZ Ceti domain is reached, hydrogen burning becomes completely unimportant.

- Element diffusion strongly modifies the shape of the chemical profile during WD evolution. In particular, before the $\mathrm{ZZ}$ Ceti stage is reached, diffusion has already smoothed out the chemical profile to such a degree that the resulting external abundance distribution does not depend on the occurrence of overshoot episodes during the thermally pulsing phase on the AGB.

Finally, the implications for the pulsational properties are:

- Models with core overshooting (OV models) exhibit a much more pronounced non-uniformity in the spacing of consecutive periods, at variance with the situation without core overshooting.

- OV models are characterized by the presence of modes which are "core trapped", i.e., modes with enhanced kinetic energy having eigenfunctions with relatively large amplitudes at the innermost, high-density regions of the models.

- Pulsational properties are insensitive to the occurrence of overshoot episodes during the thermally pulsing AGB phase. This is due to the powerful action of element diffusion during WD evolution.

- The period spectrum for our models is markedly different from that of existing computations based on the use of the trace element approximation for assessing the shape of the chemical interfaces.

- The trace element approximation need not be made in the solution of the equation of diffusive equilibrium given in Arcoragi \& Fontaine (1980), which results in smooth profiles which are closer to the results of time-dependent diffusion.

We conclude that the pulsational spectrum of massive ZZ Ceti stars as predicted by our new and improved evolutionary models turns out to be substantially different from that of previous research. In particular, because element diffusion strongly smoothes out the chemical profiles, the mode trapping caused by the outer chemical interfaces is notably diminished. As a result, the pulsational properties become very sensitive to the shape of the innermost chemical profile, i.e., the occurrence of core overshoot during the evolutionary stages prior to the WD formation. This could in principle be tested from observations of massive ZZ Ceti stars. We believe that this aspect would deserve an exploration also in the frame of less massive $\mathrm{ZZ}$ Ceti models than considered here.

In closing, we judge that the evolutionary modeling presented in this work constitutes a physically sound and solid enough frame for exploring the pulsational properties of crystallized ZZ Ceti stars. We will address this aspect in a future communication.

Acknowledgements. LGA warmly acknowledges T. Blöcker for sending us some reprints central to this work. We also thank the suggestions and comments of our referee, D. Koester, that strongly improved the original version of this work. This research was supported by the Instituto de Astrofísica de La Plata and by the UK Particle Physics and Astronomy Research Council.

\section{References}

Alexander, D. R., \& Ferguson, J. W. 1994, ApJ, 437, 879

Althaus, L. G., \& Benvenuto, O. G. 2000, MNRAS, 317, 952

Althaus, L. G., Serenelli, A. M., Córsico, A. H., \& Benvenuto, O. G. 2002, MNRAS, 330, 685

Anders, E., \& Grevesse, N. 1986, Geochim. Cosmochim. Acta, 53, 197

Angulo, C., Arnould, M., Rayet, M., et al. 1999, Nucl. Phys. A, 656, 3

Arcoragi, J.-P., \& Fontaine, G. 1980, ApJ, 242, 1208

Arnett, W. D., \& Truran, J. W. 1969, ApJ, 157, 339

Blöcker, T. 1995, A\&A, 297, 727

Bradley, P. A. 1996, ApJ, 468, 350

Bradley, P. A. 1998, ApJS, 116, 307

Bradley, P. A. 2001, ApJ, 552, 326

Brassard, P., Fontaine, G., Wesemael, F., \& Hansen, C. J. 1992a, ApJS, 80, 369

Brassard, P., Fontaine, G., Wesemael, F., \& Tassoul, M. 1992b, ApJS, 81,747

Brassard, P., Fontaine, G., Wesemael, F., Kawaler, S. D., \& Tassoul, M. 1991, ApJ, 367, 601

Brickhill, A. J. 1991, MNRAS, 251, 673

Burgers, J. M. 1969, Flow Equations for Composite Gases (New York: Academic)

Caughlan, G. R., \& Fowler, W. A. 1988, Atomic Data Nucl. Data Tables, 40, 290

Córsico, A. H., Althaus, L. G., Benvenuto, O. G., \& Serenelli, A. M. 2001, A\&A, 380, L17

Córsico, A. H., Althaus, L. G., Benvenuto, O. G., \& Serenelli, A. M. 2002, A\&A, 387, 531

D’Antona, F., \& Mazzitelli, I. 1990, ARA\&A, 28, 139

D’Antona, F., \& Mazzitelli, I. 1996, ApJ, 470, 1093

Fontaine, G., \& Michaud, G. 1979, ApJ, 231, 826

Freytag, B., Ludwig, H.-G., \& Steffen, M. 1996, A\&A, 313, 497

Gautschy, A., \& Althaus, L. G. 2002, A\&A, 382, 141

Gautschy, A., \& Saio, H. 1995, ARA\&A, 33, 75

Gautschy, A., \& Saio, H. 1996, ARA\&A, 34, 551

Gautschy, A., Ludwig, H., \& Freytag, B. 1996, A\&A, 311, 493

Goldreich, P., \& Wu, Y. 1999, ApJ, 511, 904

Grossman, S. A., \& Taam, R. E. 1996, MNRAS, 283, 1165

Grossman, S. A., Narayan, R., \& Arnett, D. 1993, ApJ, 407, 284

Herwig, F. 2000, A\&A, 360, 952

Herwig, F., Blöcker, T., Langer, N., \& Driebe, T. 1999, A\&A, 349, L5

Herwig, F., Blöcker, T., Schönberner, D., \& El Eid, M. 1997, A\&A, 324, L81

Iben, I. Jr., \& MacDonald, J. 1985, ApJ, 296, 540

Iben, I. Jr., \& MacDonald, J. 1986, ApJ, 301, 164 
Iben, I. Jr., \& Renzini, A. 1983, ARA\&A, 21, 271

Iglesias, C. A., \& Rogers, F. J. 1996, ApJ, 464, 943

Kanaan, A., Kepler, S. O., Giovannini, O., \& Diaz, M. 1992, ApJ, 390, L89

Kawaler, S. D., Winget, D. E., \& Hansen, C. J. 1985, ApJ, 295, 547

Kippenhahn, R., Weigert, A., \& Hofmeister, E. 1967, Methods in Computational Physics 7 (New York: Academic), 129

Magni, G., \& Mazzitelli, I. 1979, A\&A, 72, 134

Mazzitelli, I., D’Antona, F., \& Ventura, P. 1999, A\&A, 348, 846

Montgomery, M. H., \& Winget, D. E. 1999, ApJ, 526, 976

Osaki, Y., \& Hansen, C. J. 1973, ApJ, 185, 277

Pfeiffer, B., Vauclair, G., Dolez, N., et al. 1996, A\&A, 314, 182

Sackmann, I. J., \& Boothroyd, A. I. 1992, ApJ, 392, L71

Saio, H., \& Cox, J. P. 1980, ApJ, 236, 549

Straniero, O., Chieffi, A., \& Limongi, M. 1997, ApJ, 478, 332
Straniero, O., Domínguez, I., Imbriani, G., \& Piersanti, L. 2003, ApJ, 583,878

Tassoul, M., Fontaine, G., \& Winget, D. E. 1990, ApJS, 72, 335

Unno, W., Osaki, Y., Ando, H., Saio, H., \& Shibahashi, H. 1989, Nonradial Oscillations of Stars (University of Tokyo Press, 2nd ed.

Vassiliadis, E., \& Wood, P. R. 1993, ApJ, 413, 641

Ventura, P., D’Antona, F., \& Mazzitelli, I. 1999, ApJ, 524, L111

Ventura, P., Zeppieri, A., Mazzitelli, I., \& D’Antona, F. 1998, A\&A, 334, 953

Wagenhuber, J., \& Groenewegen, M. A. T. 1998, A\&A, 340, 183

Winget, D. E., Van Horn, H. M., \& Hansen, C. J. 1981, ApJ, 245, L33

Winget, D. E., Kepler, S. O., Kanaan, A., Montgomery, M. H., \& Giovannini, O. 1997, ApJ, 487, L191 